\title{
Mycobacterial HelD is a nucleic acids-clearing factor for RNA
}

\author{
polymerase
}

Tomáš Kouba ${ }^{* * \#, ~ T o m a ́ s ̌ ~ K o v a l ’ b *, ~ P e t r a ~ S u d z i n o v a ́ c *, ~ J i r ̌ i ́ ~ P o s p i ́ s ̌ i l c, ~ B a r b o r a ~ B r e z o v s k a ́ c, ~ J a r m i l a ~}$ Hnilicovác, Hana Šanderovác, Martina Janouškovác, Michaela Šikovác, Petr Haladac, Michal Sýkora ${ }^{d}$, Ivan Barvík ${ }^{e}$, Jiří Nováček', Mária Trundováb, Jarmila Duškováb, Tereza Skálováb, URee Chong, Katsuhiko S. Murakamig, Jan Dohnálek ${ }^{\mathrm{b \#}}$, Libor Krásnýc\#

a EMBL Grenoble, 71 Avenue des Martyrs, France

${ }^{b}$ Institute of Biotechnology of the Czech Academy of Sciences, Centre BIOCEV, Prumyslova 595, 25250 Vestec, Czech Republic

c Institute of Microbiology of the Czech Academy of Sciences, Prague, Czech Republic

d Institute of Molecular Genetics of the Czech Academy of Sciences, Prague, Czech Republic

e Charles University, Faculty of Mathematics and Physics, Institute of Physics, Prague, Czech Republic

${ }^{f}$ CEITEC, Masaryk University, Brno, Czech Republic

g Department of Biochemistry and Molecular Biology, The Center for RNA Molecular Biology, Pennsylvania State University, University Park, PA 16802, USA

\section{*Equal contribution}

\#Corresponding author: tkouba@embl.fr, dohnalek@ibt.cas.cz, krasny@biomed.cas.cz 
RNA synthesis is central to life, and RNA polymerase depends on accessory factors for recovery from stalled states and adaption to environmental changes. Here we investigated the mechanism by which a helicase-like factor HelD recycles RNA polymerase. We report a cryo-EM structure of an unprecedented complex between the Mycobacterium smegmatis RNA polymerase and HelD. The crescent-shaped HelD simultaneously penetrates deep into two RNA polymerase channels that are responsible for DNA binding and substrate delivery to the active site, thereby locking RNA polymerase in an inactive state. We show that HelD prevents non-specific interactions between RNA polymerase and DNA and dissociates transcription elongation complexes, but does not inhibit RNA polymerase binding to the initiation $\sigma$ factor. The liberated RNA polymerase can either stay dormant, sequestered by HelD, or upon HelD release, restart transcription. Our results provide insights into the architecture and regulation of the highly medically-relevant mycobacterial transcription machinery and define HeID as a clearing factor that removes undesirable nucleic acids from RNA polymerase.

A smoothly functioning transcription machinery is essential for maintaining the physiologically relevant levels of gene products and adequate changes in transcription are necessary for cell survival when the environment changes. In bacteria, transcription is core enzyme: $\alpha_{2} \beta \beta^{\prime} \omega^{1}$ ]. The RNAP core is capable of transcription elongation and termination but not initiation. To initiate, a $\sigma$ factor is required to form a holoenzyme that recognizes specific DNA sequences, promoters ${ }^{2}$. Besides the core subunits that are conserved in all 
are present in Firmicutes ${ }^{3,4}$, HelD that is present in Gram-positive bacteria ${ }^{5}$, or CarD and RbpA ${ }^{6}$ that are found in Actinobacteria. The smooth functioning of the transcription machinery then depends on concerted activities of RNAP and numerous transcription factors.

One of these factors is HelD ${ }^{5}$, a protein similar to SF1 helicases ${ }^{7}$ that associates with the RNAP core in the model gram-positive bacterium Bacillus subtilis (Bsu) where it was shown to be involved in transcriptional recycling ${ }^{8}$. HelD binds and hydrolyzes ATP and this is accompanied by conformational changes in the protein as demonstrated by SAXS experiments ${ }^{9}$. The absence of HelD from Bsu cells results in prolonged lag phase during outgrowth of stationary phase cells when diluted into fresh medium; overexpression of HelD then accelerates spore formation ${ }^{10}$. However, the structure of HelD, its binding mode to RNAP, and mechanistic details of its function are unknown.

Here, we present structural data for HelD from Mycobacterium smegmatis (Msm) in complex with the RNAP core and provide insights into its function. We solved the 3D structure of three complexes of Msm RNAP and HelD by cryogenic electron microscopy (cryo-EM). The structures represent a so far unknown type of interaction between an RNAP and a protein. Furthermore, we show that mycobacterial HelD and $\sigma^{A}$ may simultaneously bind to the RNAP core and we provide biochemical evidence showing that in addition to ATP, HelD can also hydrolyze GTP. Finally, we demonstrate that HelD can both prevent binding of the RNAP core to non-specific DNA and actively remove RNAP from stalled elongation complexes. 
Results

\section{Cryo-EM of Msm RNAP-HelD complex}

Our long-term attempts to crystalize Bsu HelD, RNAP core, or their complex failed; our cryoEM experiments with the Bsu RNAP core were not successful; also, our recent SAXS-based data for the Bsu HelD-RNAP complex were not fully conclusive. However, in coimmunoprecipitation experiments with Msm RNAP we identified MSMEG_2174, a potential homolog of Bsu HelD (Figure S1). We also solved the X-ray crystal structure of Bsu HelD Cterminal domain (CTD), which was then used as a guide for building the model of Msm HelD.

We reconstituted a complex of Msm RNAP core and Msm HelD from purified recombinant proteins (Figure S2), and froze an isolated homogenous fraction of the complex on cryo-EM grids. We collected multiple preliminary cryo-EM datasets, which allowed us to optimize the cryo-EM conditions for high-resolution three-dimensional (3D) single-particle reconstructions (Figure S3). We identified two major 3D classes (State I and State II, Figure S4) at overall resolution $\sim 3.1 \AA$ (plus one subclass at $\sim 3.6 \AA$ ), visualising almost the complete structure of HelD bound to the RNAP core in two conformations (Figure 1a,b, Movies 1,2), and one minor class (State III; Figure S4), at $3.5 \AA$, which delineates only two domains of HelD binding to the RNAP core (Figure 1c, Movie 3). The structures of States I and II share the same overall fold of HelD, with a crescent-like shape. The main body of the crescent is sitting in between the domain 2 of the RNAP $\beta$ subunit (also called $\beta$-lobe domain) and the funnel and cleft/jaw of the $\beta^{\prime}$ subunit, burying about 774 and $2608 \AA^{211}$ in State I and 1490 and $3623 \AA^{2}$ in State II of binding surface area of $\beta$ and $\beta^{\prime}$ subunits, respectively. One end of the crescent protrudes deep into the primary channel, and the other end into the secondary channel of the RNAP core. Indeed, to be able to reach both RNAP channels simultaneously, the HelD 
93 protein is markedly elongated, around $200 \AA$ along the outer edge of the virtual crescent, and

94 the two ends of the HelD protein are separated by $\sim 75 \AA$ (State II; Figure 1b).

96 which possess unique novel folds. Interestingly, the $1 \mathrm{~A}$ domain is composed of two parts (1A-

$97 \quad 1$ and $1 \mathrm{~A}-2$ ) that are separated in the primary amino acid sequence by the intervening HelD-

98 specific domain. According to the position of the HelD domains within the primary channel

99 (PCh) and active site (AS), we name State I: PCh-engaged, State II: PCh-engaged and AS-

100 interfering, and State III: PCh dis-engaged and AS-interfering (Figure 1a-c). 


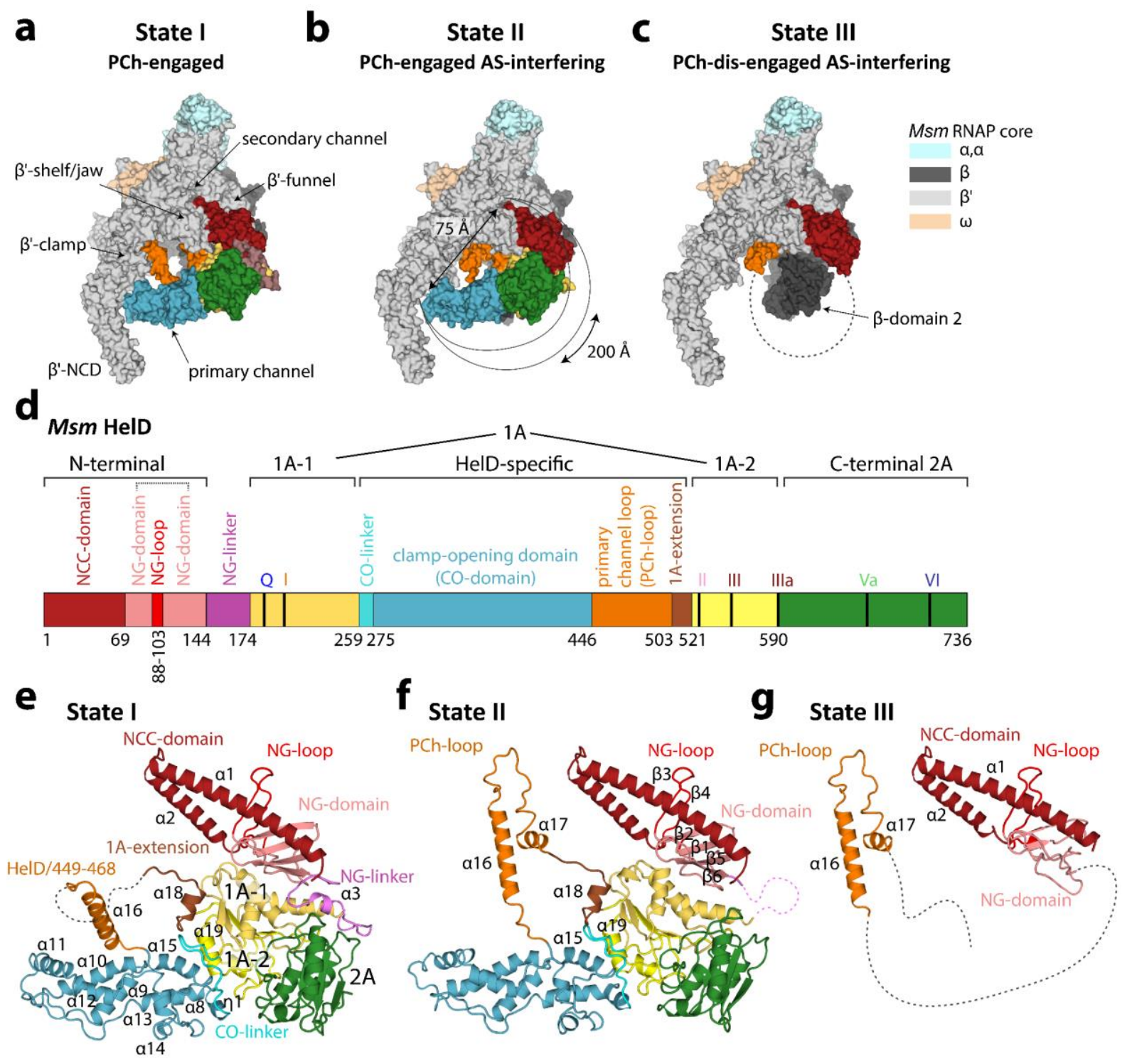

Figure 1: Cryo-EM structures of Msm HelD-RNAP complexes.

a, $\mathbf{b}$ and $\mathbf{c}$, Atomic model surface representation of three identified Msm HelD-RNAP complexes: State I - PCh-engaged, State II - PCh-engaged AS-interfering and State III - PChdis-engaged AS-interfering. When fully ordered in State I and II ( $\mathbf{a}$ and $\mathbf{b}$ ), the HelD protein (color coded as in d) forms a crescent-like shape, ends of which are protruding to the primary and secondary channel of the RNAP core. Partly ordered HelD protein in State III (c) vacates most of the RNAP primary channel. $\mathbf{d}$, Schematic linear representation of the domain structure of the HelD protein. The 1A domain (two shades of yellow) is split in aa sequence into two parts, separated by a large HelD-specific insertion (hues of blue and orange). The nucleotide binding motifs are marked as vertical thick black lines. Aa numbering $(M s m)$ is shown below. $\mathbf{e}, \mathbf{f}$ and $\mathbf{g}$, Three states of HelD as observed in $\mathbf{a}, \mathbf{b}$ and $\mathbf{c}$ color coded according to the domain structure (d), secondary structure elements are marked as in Figure S5a. 
The Msm N-terminal domain (HelD/1-144) forms an antiparallel $\alpha$-helical coiled-coil (NCC) (HelD/1-69) followed by, and packed against the four- $\beta$-strand globular (NG) domain (HelD/70-144), which contains an additional prominent protruding loop (NG-loop, residues HelD/88-103, (Figure 1d-g and Figure 2a,b). The overall N-terminal domain structure is analogous to the archetypal fold interacting with the secondary channel of RNAP present in transcription factors such as GreA or ppGpp cofactor DksA ${ }^{12-14}$. Indeed, the HelD N-terminal domain interacts tightly with the secondary channel, burying $1790 \AA^{2}$ of interaction surface, contributing largely to the HelD-RNAP interaction. Several specific hydrogen bonds and salt bridges (Table S1a) are formed between the N-terminal domain and the secondary channel, and particularly the NG-loop specifically recognises the tip of the coiled-coil (CC) motif of the $\beta^{\prime}$ funnel (Figure 2a). channel-interacting transcription factors (Figure S6); however, in contrast to the known structures of such complexes, the Msm HeID NCC is shorter and its tip does not reach into the AS (Figure S6). Indeed, a large part of the NCC is extensively packed with the NG-domain into a common hydrophobic core, thereby preventing the NCC to bind further towards the AS. The HelD NCC tip is positioned at the level of the RNAP AS $\beta^{\prime}$ bridge helix $(\mathrm{BH}), \sim 10-12 \AA$ away from $\mathrm{Mg}^{2+}$ metal $\mathrm{A}(\mathrm{MgA})$ of the $\mathrm{AS}$, and as a result, it constitutes one wall of the secondary channel pore leading to the AS. The pore itself is approximately $\sim 11 \AA$ wide (Figure $2 b$ ) and this would still allow nucleoside triphosphate (NTP) passage into the AS. On the other hand, the NCC-domain restricts the conformational freedom and induces folding of the AS trigger loop (TL, $\left.\beta^{\prime} / 1009-1028\right)$. This would likely interfere with the nucleotide addition cycle. 
a

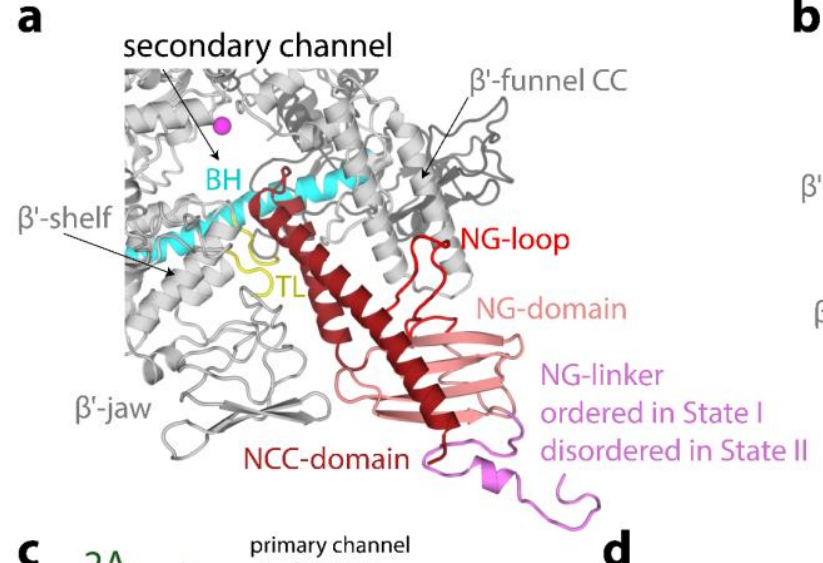

C

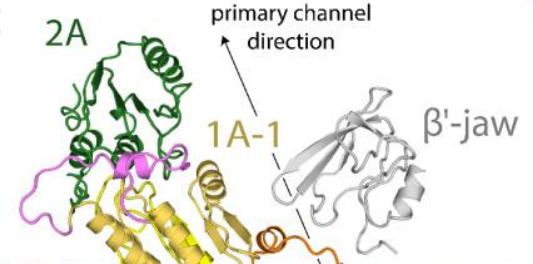

NG-linker 32 mencion

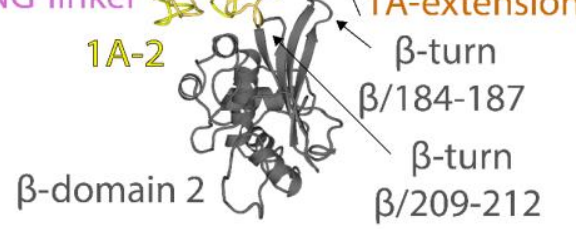

d
143
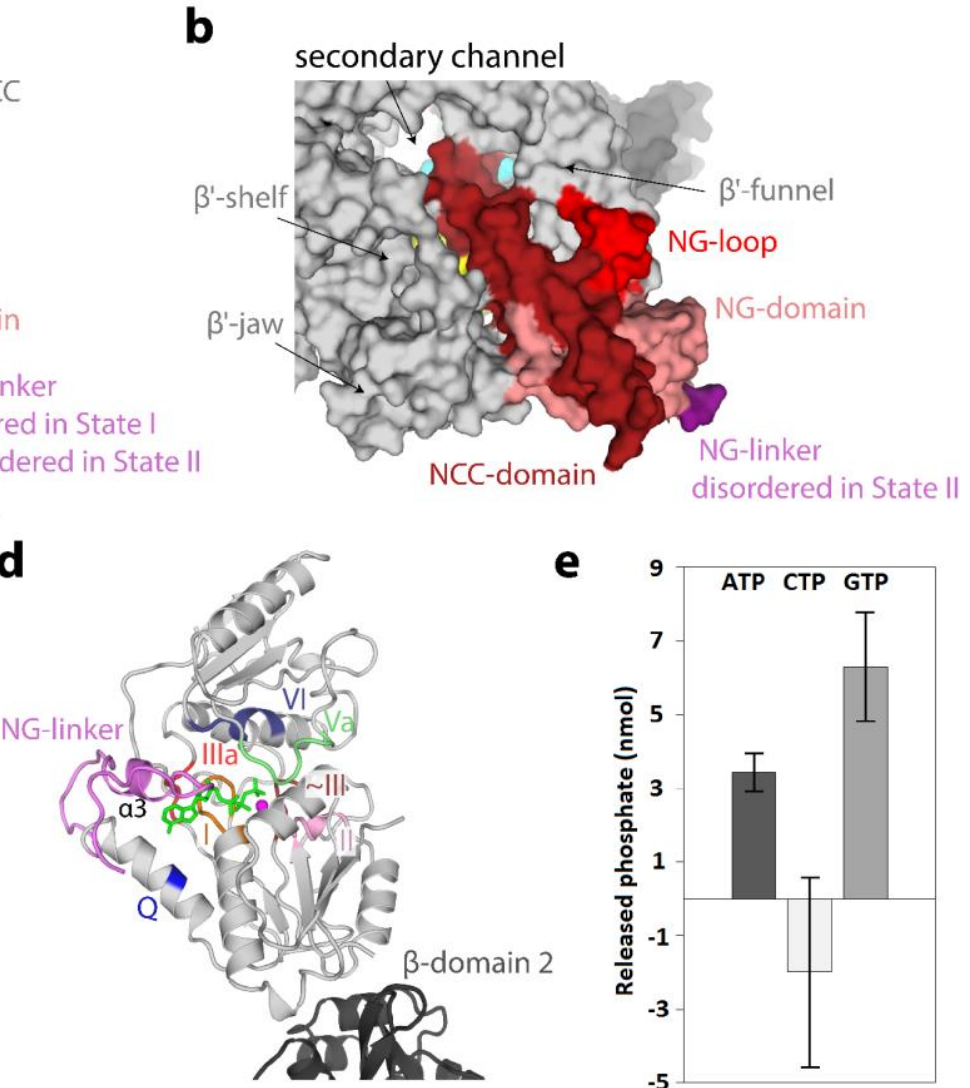

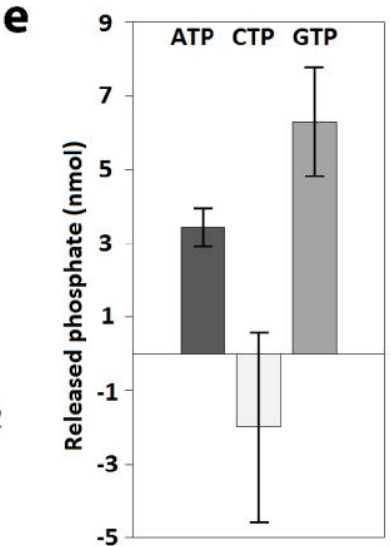

Figure 2: The HelD N-terminal domain inserts into the RNAP secondary channel; domains

\section{A-2A comprise the NTPase unit.}

$\mathbf{a}$ and $\mathbf{b}$, Ribbon (State I) and surface (State II) representation of the HelD N-terminal domain interaction with the secondary channel of RNAP core (grey). The HelD coiled-coil domain (NCC-domain, firebrick) and the distinct loop (NG-loop, red) of the HelD globular domain (NGdomain, salmon) are inserted between $\beta^{\prime}$ funnel, shelf and jaw. The NCC-domain reaches only the boundary line of the $\beta^{\prime}$ bridge helix ( $\mathrm{BH}, \mathrm{cyan}$ ) and leaves a passageway to the RNAP core active site (MgA, magenta sphere). The HelD NCC also restricts the trigger loop (TL, yellow) movement. The linker (NG-linker, violet) connects the $\mathrm{N}$-terminal domain with domain $1 \mathrm{~A}-1$. c, The two Msm HelD Rossman fold domains (1A yellow and $2 \mathrm{~A}$ green) form a canonical NTPase unit heterodimer with respect to structurally described SF1 helicases. Domain 1A tightly packs with $\beta$-domain 2 (dark grey) and its extension (brown) is clamped in-between one $\beta$-turn ( $\beta / 184-187$ ) of $\beta$-domain 2 and the tip of the $\beta^{\prime}$ subunit jaw (light grey). $\mathbf{d}$, Model 
of ATP binding to the conserved nucleotide binding site of motifs Q (blue), I (brown), II (pink), III (orange), IIla (red), Va (palegreen) and VI (deepblue). ATP (green) and $\mathrm{Mg}^{2+}$ (magenta sphere) are added based on superposition with the ternary complex of UvrD (PDB ID 2IS4). e, HelD exhibits ATPase and GTPase activities but does not hydrolyse CTP.

\section{The NTPase unit of HelD is positioned in the vicinity of the downstream section of the}

\section{primary channel}

The presented structure confirms our previous prediction ${ }^{9}$ where HelD, similarly to SF1 helicases, RapA and UvrD, contains a conserved Rossmann fold $1 \mathrm{~A}-2 \mathrm{~A}$ heterodimer. $1 \mathrm{~A}-1$ is connected with the N-terminal domain by the NG linker (HeID/145-173), which orders only in State I. $1 \mathrm{~A}-2$ is then followed by $2 \mathrm{~A}$ (Figure $1 \mathrm{~d}, \mathrm{e}, \mathrm{f}$ and $2 \mathrm{c}$ ).

The $1 \mathrm{~A}$ domain docks on the $\beta$-domain 2 where it induces small domain-orientation and conformational changes and it prolongs the wall of the downstream section of the primary channel along the axis of the virtual downstream DNA (Figure $2 \mathrm{c}$ ). The $1 \mathrm{~A}$ domain buries an area of $725 \AA^{2}$ of the interaction surface of the $\beta$-domain 2 , the binding also involves ordering of the $\beta$-turn $\beta / 209-212$ and many hydrogen bonds and salt bridges (Table S1b). In addition, the extension of the $1 \mathrm{~A}$ domain (HelD/504-521) is clamped in between the prominent $\beta$-turn $\beta / 184-187$ of the $\beta$-domain 2 and the tip of the $\beta^{\prime}$ subunit jaw, further securing the $1 \mathrm{~A}$ domain in its place.

The $1 \mathrm{~A}-2 \mathrm{~A}$ heterodimer establishes the canonical tertiary structure to form an NTPbinding pocket. Conserved residues of motifs $\mathrm{Q}, \mathrm{I}, \mathrm{II}, \sim \mathrm{II}, \mathrm{III}$, Va, and VI are then likely involved in ATP binding 7,18 (Figure 2d) while motifs and residues typical for DNA binding are missing. However, the base type specificity is not obvious from the structural data and therefore we measured nucleoside triphosphate hydrolysis activity of the isolated HelD protein. HelD showed strong hydrolysis activity of purine base nucleoside triphosphates but no activity 
towards a pyrimidine-containing counterpart (Figure 2e, S7f). We also added ATP or nonhydrolysable ATP analogue to the HelD-RNAP complex, but we were not able to visualize any NTP-bound state by cryo-EM. Indeed, the orientations of conserved HelD/Tyr589 and Arg/590 of motif IIla, which are supposed to stack and coordinate the base and phosphate groups in the canonical ATP-bound state ${ }^{19}$, are incompatible with ATP binding in the HelD NTP-free state, both in State I and II (Figure S7a). Notably, helix $\alpha 3$ of the ordered NG-linker in State I covers the putative NTP-binding pocket and partially obstructs the site entrance (Figure S7a). However, the entire linker can become disordered as seen in State II (Figure S8h), which is probably more compatible with NTP binding (see details below).

The superposition of HelD 1A-2A with similar structures of UvrD (PDB ID 2IS4) (Figure S7b,c), PcrA (PDB ID 3PJR), AdnA/B (PDB ID 6PPR) and RapA (PDB ID 6BOG) confirms that the Rossmann fold domains are packed in the canonical mutual orientation. However, unlike in bona fide SF1 helicases ${ }^{7,18}$ where SSDNA is bound in the interface cleft of the dimer by conserved motifs Ia, Ic, IV, and V (Figure S7b,c), these motifs are not conserved in HelD. Instead, HelD contains proline-rich loops in place of these motifs and a large negatively charged surface patch in the equivalent areas (Figure S7d,e). Similarly, the ssDNA binding motifs are not conserved inRapA, a functional homolog of HelD, a helicase-like protein involved in recycling of RNAP. RapA, however, binds differently to RNAP than HelD ${ }^{20}$.

The Msm HelD-specific domain is inserted into the downstream section of the RNAP primary channel

The HelD-specific insertion domain is composed of the clamp-opening domain (CO-domain, HelD/261-447) and the primary channel loop (PCh-loop, HelD/448-503) (Figure 1d, 3a,b). The CO-domain is an extended, mostly $\alpha$-helical, and completely novel fold with no structure 
homologs (Figure S5b). On one side, the CO-domain packs against the $1 \mathrm{~A}$ domain helix $\alpha 19$ and $\beta$-turn HelD/561-564. Additionally, the CO-1A interaction is stabilised by the CO-linker (HelD/259-275), which connects the two domains. In State I, the other side of the CO-domain, the CO-tip, butts against the three-stranded sheet of the $\beta^{\prime}$ non-conserved domain (NCD) and an $\alpha$-helix $\left(\beta^{\prime} / 122-133\right)$ of the $\beta^{\prime}$ clamp just preceding it (Figure 3a). The only significant ordered part of the PCh-loop in State I, the protruding helix $\alpha 16$ (HelD/451-468), is erected against the $\beta^{\prime}$ three-stranded sheet $\left(\beta^{\prime} / 1164-1210\right)$ and the $\alpha 16$ tip locks behind the helixturn-helix motif $\beta^{\prime} / 271-304$ by HelD/Tyr466. Altogether, the $\alpha 16$ interaction with the $\beta^{\prime}$ clamp might be helping the CO-domain insertion into the primary channel. In State II, the CO-domain fold alters and the PCh-loop completely refolds. The CO-tip shifts towards the $\beta^{\prime}$ clamp coiledcoil domain $\left(\beta^{\prime}-C C\right)$ domain and reaches the peptide $\beta^{\prime} / 387-389$ of the rudder (Figure $\left.3 b\right)$. The PCh-loop protruding helix $\alpha 16$ refolds ( $\alpha 16$ register slightly shifts towards the C-terminus of HelD) and dis-engages with the $\beta^{\prime}$ three-stranded sheet ( $\left.\beta^{\prime} / 1164-1210\right)$, and the whole PChloop orders towards the AS (see next section). Correspondingly, the two insertion modes of the CO-domain and PCh-loop into the primary channel force the $\beta^{\prime}$ clamp domain to swing out into two distinct positions (see details below). 

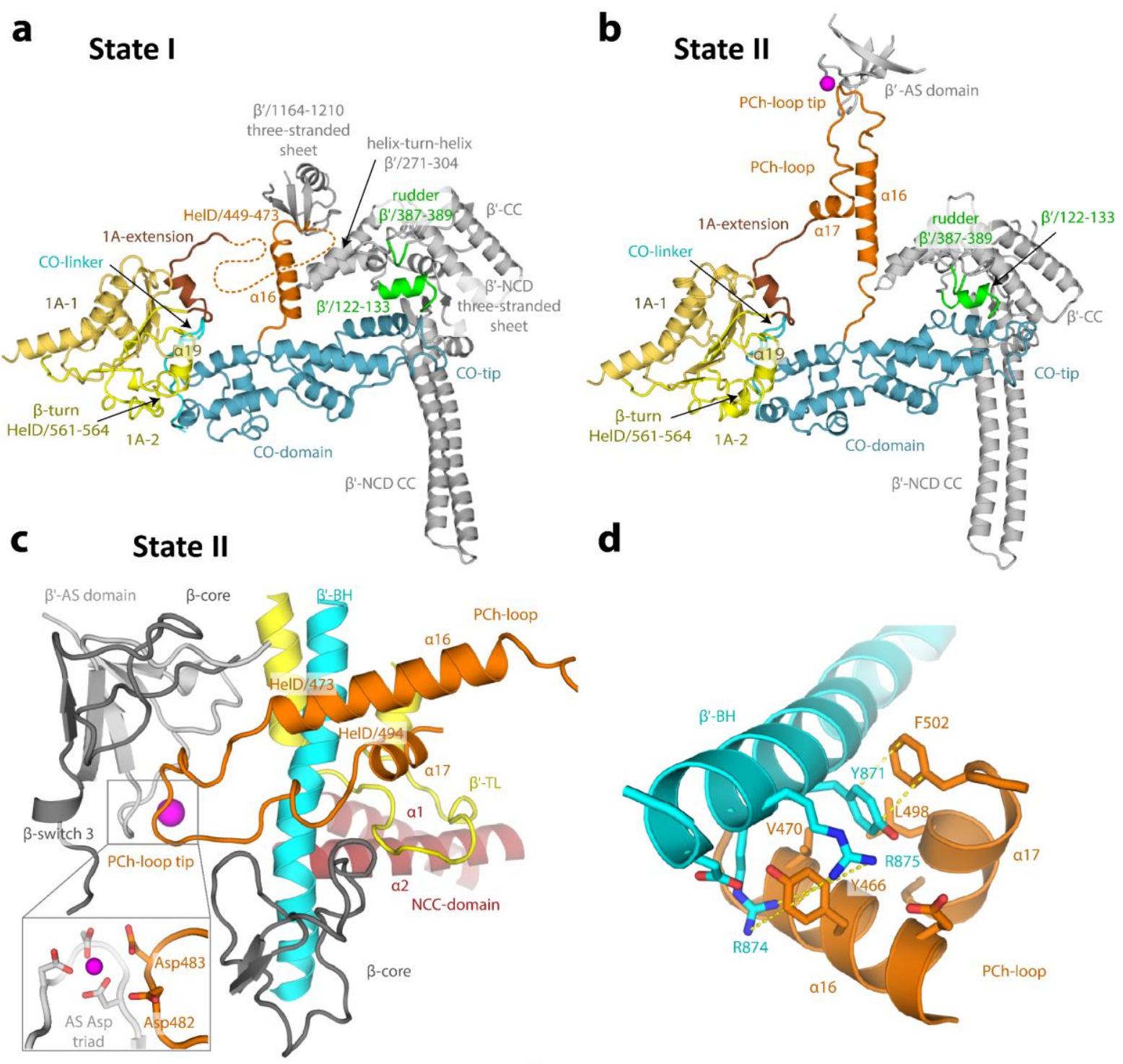

e
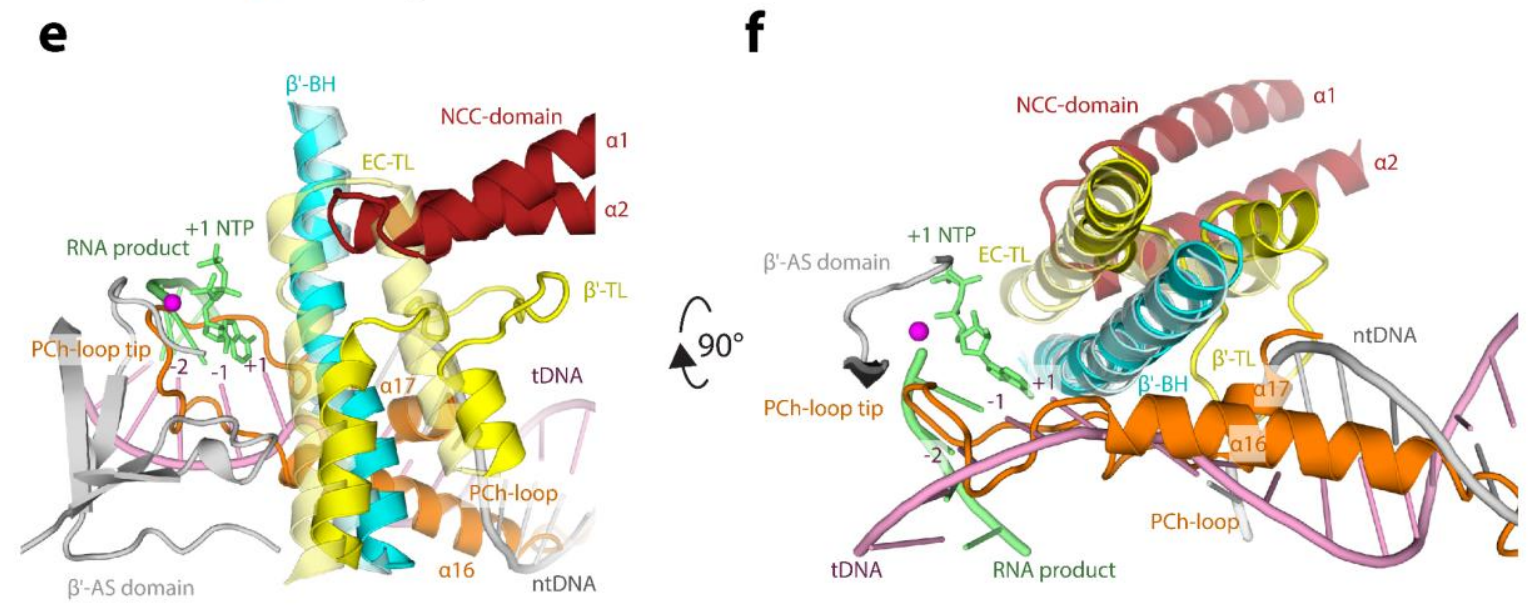

Figure 3: The Msm HelD-specific domain interactions with the RNAP primary channel.

$\mathbf{a}$ and $\mathbf{b}$, Ribbon representation of the HelD-specific domain inserting into the RNAP primary channel in State I (a) and State II (b). In State I (a), the clamp-opening (CO, blue) HelD-specific 
domain is projected from the HelD 1A domain (yellow) towards the $\beta^{\prime}$ clamp (grey). At one end, the $\mathrm{CO}$ is bonded to the $1 \mathrm{~A}$ domain by the CO-linker (cyan), and stabilised by $\beta$-turn 561563 and $\alpha 19$ (yellow). On the other end, the CO-domain tip abuts towards the $\beta^{\prime}$ NCD threestranded sheet. Concomitantly, the HelD helix $\alpha 16$ (part of peptide HelD/449-473, orange) butts against the $\beta^{\prime} / 1164-1210$ three-stranded sheet. The connection between $\alpha 16$ and the $1 \mathrm{~A}$-extension is disordered (dotted line). In State II (b), The CO interaction with the $1 \mathrm{~A}$ domain remains similar to State I (a). The CO-domain tip, however, shifts towards the $\beta^{\prime}$ rudder (green) and $\beta^{\prime} / 122-133 \alpha$-helix. Concomitantly, the HelD PCh-loop (orange) folds towards the active site (MgA, magenta sphere) and folds back towards the $1 \mathrm{~A}$-extension (brick) and $1 \mathrm{~A}$ domain. c, The PCh-loop folds into the RNAP active site. The HelD loop 473-494 and the two adjacent $\alpha$-helices ( $\alpha 16$ and $\alpha 17$, orange) fold alongside the RNAP bridge helix (BH, cyan) towards the RNAP active site and HelD/Asp482 directly contacts the MgA (magenta sphere, details in inset). The RNAP trigger loop (TL, yellow) is restricted and folded between the HelD PCh-loop helix $\alpha 17$, the HelD NCC-domain (ruby), $\beta^{\prime}$ BH and the $\beta$ core domain (dark grey). $d$, Detail of the $\mathrm{BH}$ interaction with HelD $\alpha 16$ and $\alpha 17$. BH $\beta^{\prime} /$ Arg874 and 875 sandwich HelD/Tyr466, and $\beta^{\prime} /$ Tyr871 stacks on HelD/Phe502. The hydrophobic interactions are marked by yellow dotted line. $\mathbf{e}$ and $\mathbf{f}$, The HelD PCh-loop binding in the active site chamber is mutually exclusive with the presence of the transcription bubble. Two perpendicular views of superposition of the Tt RNAP elongation complex (PDB ID 205J, pale colors) and HelD State II (solid colors) are shown. The folded TL in pre-translocated EC would sterically clash with the HelD NCC-domain. The HelD PCh-loop tip would sterically clash with RNA/DNA hybrid at positions +1 to -2 , and the HelD $\alpha 16$ and $\alpha 17$ helices would clash with downstream DNA duplex. Color code as in $\mathbf{3 c}$, template DNA in pink, non-template DNA in grey, product RNA and incoming NTP at position +1 in green.

\section{The HelD PCh-loop is able to fold into the RNAP active site}

In the cryo-EM map of the AS-interfering State II, high-resolution density is present for the entire register of the PCh-loop, which is folded in the AS cavity of RNAP (Figure 3c,d and S3f). The folding of the PCh-loop in-between the walls of the AS chamber is also compatible with the regular open form of the RNAP core as observed in State III.

In comparison to State I, in State II the protruding helix $\alpha 16$ refolds, the helix register shifts to residues 455-472, and together with a newly folded helix $\alpha 17$ (HelD/495-500) they tightly pack with the second half of the $\beta^{\prime}$ BH (Figure 3d). In detail, BH $\beta^{\prime} / \operatorname{Arg} 874$ and 875 sandwich $\alpha 16 \mathrm{HelD} /$ Tyr466 and, cooperatively, BH $\beta^{\prime} /$ Tyr871 stacks on HelD/Phe502 and is inserted into a hydrophobic pocket formed by HelD/Tyr466, Ala467, Val470, and Leu498. The 
rest of the PCh-loop (HelD/473-494) specifically wedges into the AS cavity (Table S1c), contributes to the AS-interfering loop stability by a salt bridge with the side chain of - a small hydrophobic core formed by the HelD/Val475, Leu480, and Leu488 side chains and an intra-chain ion-pair HelD/Arg477-Asp491, with HelD/Arg477 leaning against $\beta /$ Pro483.

As a result of the PCh-loop folding into the primary channel and HeID NCC folding in the secondary channel, the NCC tip and the tip of the PCh-loop are brought close together (the distance is only $17 \AA$ ). This also restricts the trigger loop, which is therefore partially folded in the space between $\mathrm{BH}, \mathrm{HelD} \alpha 2$ and $\alpha 17$, the peptide between $\alpha 17$ and $\alpha 18$, and the peptide of $\beta /$ Ile182-Glu187. In summary, the PCh-loop seems to interfere with the AS cavity so that it is not compatible with the NTP addition cycle. Moreover, the superposition with a model of Thermus thermophilus (Tt) RNAP EC (PDB ID 2O5J) (Figure 3e,f) suggests that the whole PCh-loop would be in steric clash with the dwDNA duplex and the RNA/DNA hybrid in the AS as far as position -2. A parallel can be drawn between the presence of the PCh-loop in State II and the so called DNA-mimicking loop of Poll ${ }^{21}$, which also occupies the AS chamber transcription bubble in RNAP. 
Superposition based on the $\beta$-core region ( $\beta / 430-738$ ) of the Msm RNAP core (PDB ID 6F6W), elongation complex (EC, based on PDB ID 205J) and States I-III enables analyses of global differences of the three observed structural states (Figure S8). In all States, when compared to the model of EC structure, probably just the insertion of the HeID N-terminal domain into the secondary channel slightly alters the position of the $\beta^{\prime}$-jaw/shelf and $\beta$-domain 2 (Figure S8g) and this alteration may weaken interaction with dwDNA, reminiscent of TraR (a distant

The major change between the States is the interplay between the refolding of the PCh-loop and the CO-domain position in the primary channel. In State III, solely the PChloop's tight contact with the AS stabilizes a very open form of RNAP (Figure S8a,b,f), 33 $\AA$ at the narrowest point of the primary channel (measured by the distance of the C $\alpha$ atoms of $\beta /$ Lys273 and $\beta^{\prime} /$ Lys123), comparable to the structures Core2 and Holo2 ${ }^{1}$ (32.2 and $33.6 \AA$, respectively). In State I, the PCh-loop's interaction with $\beta^{\prime}$ helix-turn-helix and three-stranded sheet, and the CO-domain insertion into the primary channel make the opening of the RNAP clamp ( $\sim 35 \AA$; Figure S8a,b) slightly wider than the extremely open forms of the Lipiarmycin(PDB ID 6FBV ${ }^{23}$ ) and Fidaxomicin-locked (PDB ID 6C06 ${ }^{24}$ ) RNAPs (34.2 and $33.6 \AA$, respectively ${ }^{1}$ ). In State II (Figure S8e), while the CO-domain still inserted, the PCh-loop abolishes the $\beta^{\prime}$ contact and folds in the AS instead, and this forces the $\beta^{\prime}$-clamp ( $\left.\beta^{\prime} / 1-406\right)$ to rotate with respect to the remaining parts of the complex so that the $\beta^{\prime}-\mathrm{NCD} C \mathrm{C}$ tip opens further away from the juxtaposed $\beta$-domain 2 but at the same time the $\beta^{\prime}$-rudder, $\beta^{\prime}$-CC and adjacent secondary elements move about $11 \AA$ closer to the tip of the HelD CO-domain. The 
RNAP clamp is therefore splayed by unprecedented $45 \AA$ (Figure S8b) and this likely facilitates DNA release.

The next major difference are the $\beta$-domain 2 and CO-domain adjustments upon change of the $1 \mathrm{~A}-2 \mathrm{~A}$ heterodimer (Figure S8e). The mutual orientation of $1 \mathrm{~A}$ and $2 \mathrm{~A}$ domains between States I and II is almost preserved, although with much poorer density for $2 \mathrm{~A}$ in State II. This most likely stems from the more pronounced mobility of $2 \mathrm{~A}$, possibly linked with the lack of stabilization by the unfolded NG-linker in State II. The 2A relaxation allows movement of $1 \mathrm{~A}$ in respect to the $\mathrm{N}$-terminal domain ( $\sim 3^{\circ}$ difference measured by HelD $\alpha 1$ and $\left.\alpha 5\right)$ and a concomitant shift of both the $\beta$-domain 2 and CO-domain (Figure S8e). In detail, this global change is accompanied by a shift and changes in the secondary structure of HelD/230-252 within the 1A domain (largest shift about $9.3 \AA$ for Val245). Helix $\alpha 6$ is extended and helix $\alpha 7$ is formed in State II (Figure S5a) and 1A-extension shifted. State I interactions between $\alpha 6$ and the NTPase site, and $\alpha 6$ and the NG-linker that are NTP-binding prohibitive, are broken in State II and the NTPase site of HelD becomes wide open (NTP-binding permissive; Figure S8h). Although this change makes the NTPase site accessible for NTPs, additional conformational changes are still required for NTP accommodation.

Finally, HelD binding in States I and II also leads to opening of the RNA exit channel between the $\beta$-flap and $\beta^{\prime}$-lid and $\beta^{\prime}$-Zn-finger by about $15 \AA$ and $21 \AA$, respectively (Figure S8c,d). State III keeps the channel still rather open by about $12 \AA$. This is expected to contribute to RNA release.

\section{HelD clears the RNAP primary channel}

326 The position of the HelD CO-domain in the primary channel of RNAP suggests that HelD may prevent non-specific interactions between the RNAP core and DNA. To test this, we 
performed electrophoretic mobility shift assay (EMSA) with RNAP and a fragment of mycobacterial DNA in the presence/absence of HelD. Figure $4 a, b, c$ shows that HelD significantly abolishes the nonspecific binding of the RNAP core to DNA.

Moreover, we speculated that HelD might not only prevent DNA binding, but also actively disassemble stalled ECs. Stalled ECs (due to $e$. $g$. damaged DNA) are obstacles for both the coupled transcription-translation machinery ${ }^{25,26}$ and also for replication ${ }^{27}$, with potentially deleterious consequences if not removed. To test the ability of HelD to rescue stalled RNAP, we assembled ECs with the RNAP core on a DNA-RNA scaffold and challenged them with HelD in the presence/absence of NTPs (Figure 4d). HelD then, relative to mock treatment, was able to disassemble stalled ECs (Figure 4e). This process, interestingly, appeared to be independent of ATP or GTP.

\section{HelD, $\sigma^{A}$ and RbpA can simultaneously bind RNAP core}

Next, we tested the mode of binding of HelD to RNAP, asking whether the HelD-RNAP complex is compatible with the presence of other factors. Immunoprecipitation (IP) and Western blot experiments with FLAG-tagged Msm RNAP revealed the presence of HelD and $\sigma^{A}$ (Figure 4f,g); FLAG-tagged $M s m \sigma^{A}$ pulled down the RNAP core and HelD; FLAG-tagged HelD pulled down the RNAP core and $\sigma^{A}$. These results suggested but not proved that HelD, $\sigma^{A}$, and RNAP could be in one complex. Alternatively, HelD and $\sigma^{A}$ could bind each other independently of RNAP. To decide between the two possibilities, we first pulled down FLAG-tagged HelD and associated proteins and from this mixture we subsequently pulled down FLAG-tagged $\sigma^{\mathrm{A}}$. Figure S9 shows the presence of HelD and RNAP in the second pull-down, demonstrating that these proteins can coexist in one complex. Additionally, RbpA, albeit in low amounts, was also present in the HelD-immunoprecipitated complex and RbpA-FLAG pulled down RNAP with $\sigma^{A}$ 
a

b

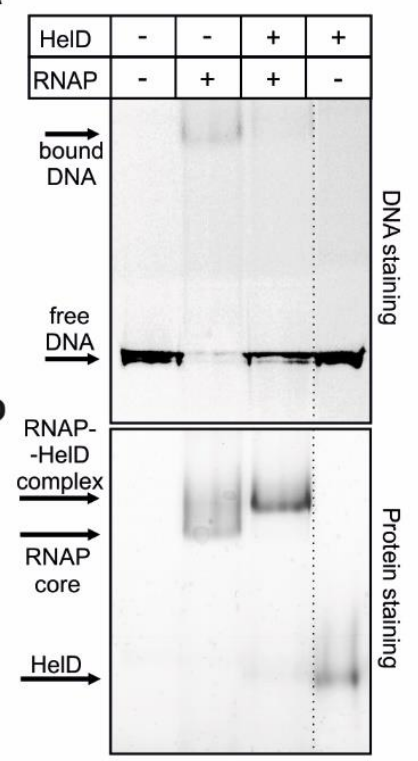

c

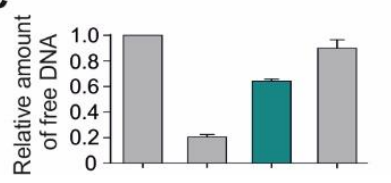

d
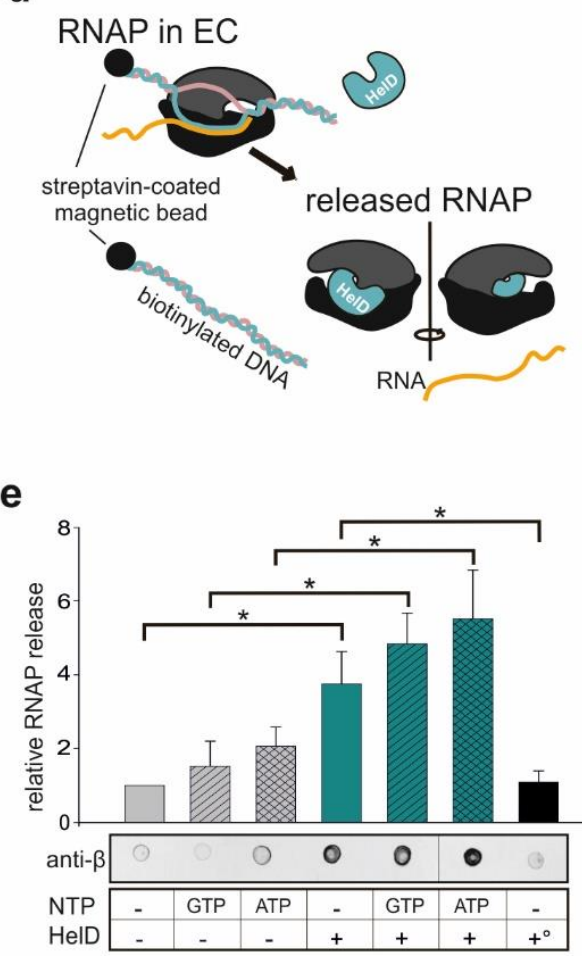

f
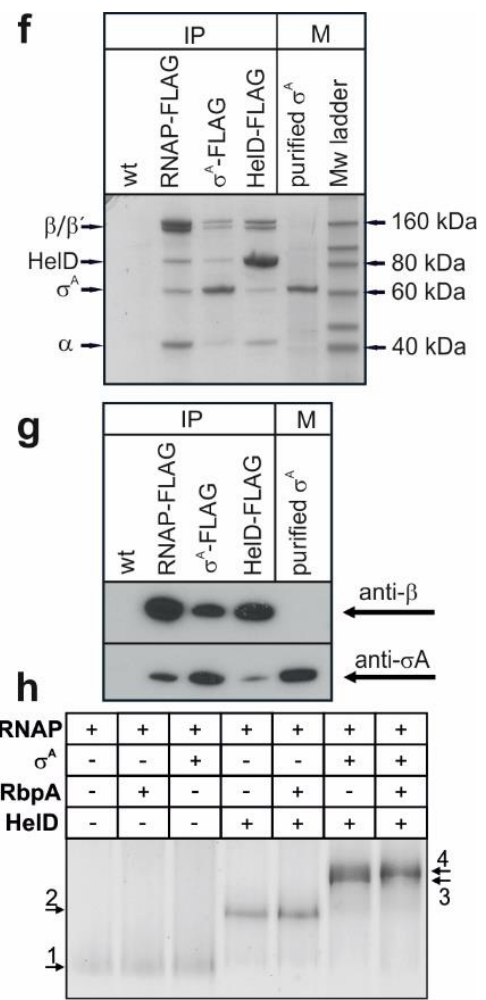

Figure 4: Binding of Msm HelD to RNAP and its effects on DNA-RNAP interactions.

a, DNA binding to RNAP - EMSA - binding of $300 \mathrm{bp}$ DNA to the Msm RNAP core and the effect of HelD. $\mathbf{b}$, the same gel as above but stained for proteins. The dotted line shows where the gel was electronically assembled. c, quantitation of EMSA - the bars here (the amount of unshifted DNA) and in e are averages from at least three independent experiments, the error bars show \pm SD. d, EC disassembly - scheme: ECs were assembled on DNA:RNA scaffolds and challenged with HelD and/or NTPs. RNAP released into buffer was quantitated by Western dot blots. e, Quantitation of EC disassembly experiments. Representative primary data are shown below the graph. Presence/absence of individual components is indicated. $+^{\circ}$ indicates heat-inactivated HelD. The statistical significance in e for the indicated combinations was $p<0.05$ (asterisks). $\mathbf{f}$, SDS PAGE of immunoprecipitations of Msm RNAP $(\beta), \sigma^{A}$, and HelD. All proteins were FLAG fusions, the antibody was anti-FLAG. Wt, a strain without any FLAG fusion. The identity of the bands was confirmed by mass spectrometry. IP, immunoprecipitation; $M$, markers. g, Western blot of IPs of FLAG-tagged Msm RNAP $(\beta), \sigma^{A}$, and HelD. Antibodies against RNAP $\beta$ and $\sigma^{A}$ were used to detect the presence of proteins in complexes. $M$, marker. 
h, In vitro protein interactions - EMSA. Proteins were detected by Simply blue SafeStain. In all cases, RNAP was first reconstituted with HelD and then with RbpA and/or $\sigma^{A}$. A small, but reproducible shift was observed after addition of both RbpA and $\sigma^{A}$ to RNAP-HelD, indicating the presence of all proteins in one complex. Numbered arrows indicate complexes with different protein composition (determined by mass spectrometry). In some cases, complexes with different protein composition displayed the same migration in the gel: 1. RNAP, RNAPRbpA; RNAP- $\sigma^{A}$; 2. RNAP-HelD, RNAP-HelD-RbpA; 3. RNAP-HelD- $\sigma^{A}$; 4. RNAP-HelD- $\sigma^{A}-R b p A$.

\section{Discussion}

This study describes a structurally unique complex between Msm RNAP and the HelD protein, defines its DNA-clearing activity, and outlines its role in transcription.

Previous biochemical studies used HelD from Bsu, which is only $21 \%$ identical with the Msm homologue. Selected sequence homologs of Msm HelD are shown in Figure S12, revealing two main differences between Msm (Actinobacteria) and Bsu (Firmicutes). The first marked difference is the absence of $\sim 30$ aa from the N-terminal NCC-domain region in Msm HelD. This is consistent with the Bsu HelD NCC-domain protruding much deeper into the RNAP secondary channel and even overlapping with the AS (See accompanying papers Newing et al., 2020; Pei et al., 2020). The other difference is in the HelD-specific region where Bsu HelD completely lacks the PCh-loop. On the other hand, the organisation of the $1 \mathrm{~A}-1$ and $1 \mathrm{~A}-2$ split followed by the complete $2 A$ domain is maintained (Figure $1 d, e, f)$.

Based on the structural and functional data we propose a role for Msm HelD in transcription (Figure 5). We envisage that upon transcription termination when RNAP fails to dissociate from nucleic acids ${ }^{28}$, or in the event of stalled elongation, Msm HelD first interacts with RNAP by its N-terminal domain, likely competing for binding to the secondary channel with GreA-like factors. This initial HelD binding induces changes in $\beta$-domain 2 and $\beta^{\prime}$ jaw/shelf (Figure S8g), possibly leading to destabilisation of DNA in the primary channel. The trigger loop is conformationally locked. Subsequently, the CO-domain and PCh-loop approach 
the primary channel. The PCh-loop, which is probably flexible in the RNAP-unbound state, folds partially upon binding RNAP (captured in State I) and then it penetrates deep into the primary channel, fully folds, and binds to the AS (captured in States II and III). The CO-domain interactions with $\beta^{\prime}$ then secure the primary channel wide open (Figure $S 8 a, b$ ). At the same time, the RNA exit channel dilates (Figure S8c,d). All these processes lead to expulsion of any contents of the AS (compare states within Figure S8a).

We note that neither HelD loading onto RNAP nor RNAP clamp opening nor EC disassembly are dependent on NTP hydrolysis. Energy from NTP hydrolysis is probably required to release HelD from its tight contact with RNAP. Free energy corresponding to ATP hydrolysis under physiological conditions in cells is around $-50 \mathrm{~kJ} / \mathrm{mol}^{29}$. This is comparable to the estimated desolvation energy of the HelD:RNAP core interaction of $-33.5 \mathrm{~kJ} / \mathrm{mol}\left(\Delta^{\mathrm{i}} \mathrm{G}\right)$ for State I and $-57 \mathrm{~kJ} / \mathrm{mol}$ for State II. However, States I and II are not fully compatible with canonical NTP binding in the HelD NTPase unit. It remains to be answered which structural changes are required to actually enable NTP binding and hydrolysis.

Interestingly, HelD, $\sigma^{A}$, and RbpA can co-occur on RNAP (Figures $4 \mathrm{~h}$ and S9-11). This differs from Bsu where simultaneous HelD and $\sigma^{A}$ binding has not been detected ${ }^{8}$. Regardless of the exact mutual $\sigma^{A}, \operatorname{RbpA}$ and HelD positions, RNAP must subsequently assume a conformation that is compatible with promoter DNA binding and transcription initiation.

To summarize, HelD clears RNAP of undesirable nucleic acids, which likely contributes to the smooth functioning of the transcription machinery. Furthermore, it is conceivable that HelD may also function similarly to $6 \mathrm{~S} \mathrm{RNA}^{30}$ or $\mathrm{Ms}^{31}$, keeping RNAP in an inactive state under unfavourable conditions. This stored RNAP then accelerates restart of gene expression when conditions improve. 
seen e.g. in the action of fidaxomicin towards M. tuberculosis RNAP in complex with RbpA ${ }^{24}$.

a stalled EC

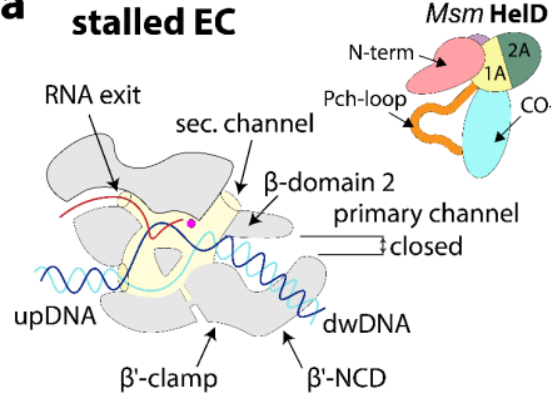

b

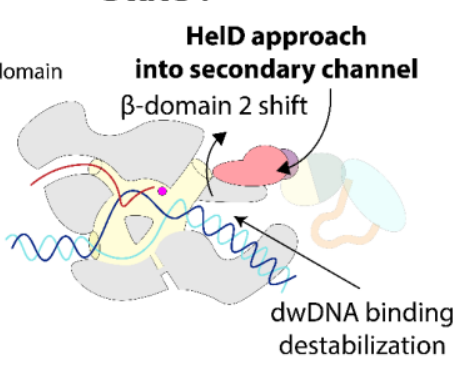

\section{C based on State I}

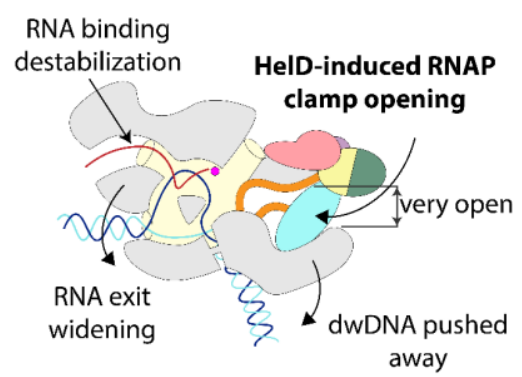

f

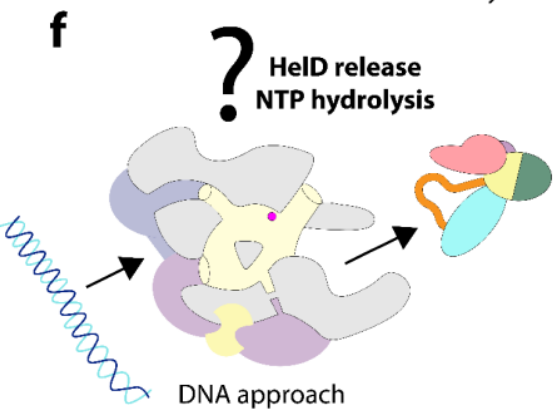

d

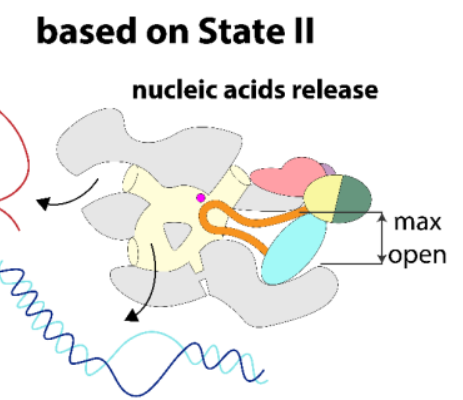

g

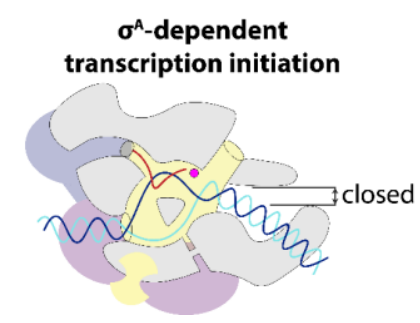

e based on State III

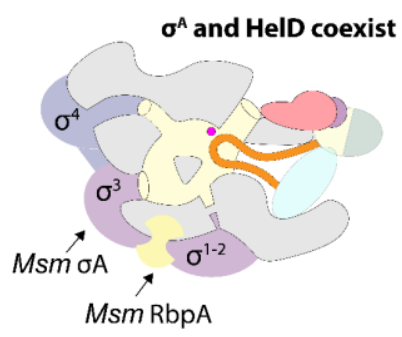




\section{References}

4401 Kouba, T. et al. The Core and Holoenzyme Forms of RNA Polymerase from Mycobacterium smegmatis. J Bacteriol 201, doi:10.1128/JB.00583-18 (2019).

Paget, M. S. Bacterial Sigma Factors and Anti-Sigma Factors: Structure, Function and Distribution. Biomolecules 5, 1245-1265, doi:10.3390/biom5031245 (2015). polymerase binding properties of the delta factor of Bacillus subtilis. J Biol Chem 274, 1595315958, doi:10.1074/jbc.274.22.15953 (1999).

(2019).

7 Fairman-Williams, M. E., Guenther, U. P. \& Jankowsky, E. SF1 and SF2 helicases: family Keller, A. N. et al. epsilon, a new subunit of RNA polymerase found in gram-positive bacteria. J Bacteriol 196, 3622-3632, doi:10.1128/JB.02020-14 (2014).

matters. Curr Opin Struct Biol 20, 313-324, doi:10.1016/j.sbi.2010.03.011 (2010)

8 Wiedermannova, J. et al. Characterization of HelD, an interacting partner of RNA polymerase from Bacillus subtilis. Nucleic Acids Res 42, 5151-5163, doi:10.1093/nar/gku113 (2014).

9 Koval, T. et al. Domain structure of HelD, an interaction partner of Bacillus subtilis RNA polymerase. FEBS Lett 593, 996-1005, doi:10.1002/1873-3468.13385 (2019).

10 Meeske, A. J. et al. High-Throughput Genetic Screens Identify a Large and Diverse Collection of New Sporulation Genes in Bacillus subtilis. PLoS Biol 14, e1002341, doi:10.1371/journal.pbio.1002341 (2016).

11 Krissinel, E. \& Henrick, K. Inference of macromolecular assemblies from crystalline state. J Mol Biol 372, 774-797, doi:10.1016/j.jmb.2007.05.022 (2007).

12 Ross, W. et al. ppGpp Binding to a Site at the RNAP-DksA Interface Accounts for Its Dramatic Effects on Transcription Initiation during the Stringent Response. Mol Cell 62, 811-823, doi:10.1016/j.molcel.2016.04.029 (2016).

13 Abdelkareem, M. et al. Structural Basis of Transcription: RNA Polymerase Backtracking and Its Reactivation. Mol Cell 75, 298-309 e294, doi:10.1016/j.molcel.2019.04.029 (2019).

14 Molodtsov, V. et al. Allosteric Effector ppGpp Potentiates the Inhibition of Transcript Initiation by DksA. Mol Cell 69, 828-839 e825, doi:10.1016/j.molcel.2018.01.035 (2018).

15 Laptenko, O., Lee, J., Lomakin, I. \& Borukhov, S. Transcript cleavage factors GreA and GreB act as transient catalytic components of RNA polymerase. EMBO J 22, 6322-6334, doi:10.1093/emboj/cdg610 (2003).

16 Perederina, A. et al. Regulation through the secondary channel--structural framework for ppGpp-DksA synergism during transcription. Cell 118, 297-309, doi:10.1016/j.cell.2004.06.030 (2004).

17 Sosunova, E. et al. Donation of catalytic residues to RNA polymerase active center by transcription factor Gre. Proc Natl Acad Sci U S A 100, 15469-15474, doi:10.1073/pnas.2536698100 (2003).

18 Raney, K. D., Byrd, A. K. \& Aarattuthodiyil, S. Structure and Mechanisms of SF1 DNA Helicases. Adv Exp Med Biol 973, E1, doi:10.1007/978-1-4614-5037-5_14 (2013).

19 Lee, J. Y. \& Yang, W. UvrD helicase unwinds DNA one base pair at a time by a two-part power stroke. Cell 127, 1349-1360, doi:10.1016/j.cell.2006.10.049 (2006).

20 Liu, B., Zuo, Y. \& Steitz, T. A. Structural basis for transcription reactivation by RapA. Proc Natl Acad Sci U S A 112, 2006-2010, doi:10.1073/pnas.1417152112 (2015). 
21 Tafur, L. et al. Molecular Structures of Transcribing RNA Polymerase I. Mol Cell 64, 11351143, doi:10.1016/j.molcel.2016.11.013 (2016). Chen, J. et al. Stepwise Promoter Melting by Bacterial RNA Polymerase. Mol Cell 78, 275-288 e276, doi:10.1016/j.molcel.2020.02.017 (2020). Lin, W. et al. Structural Basis of Transcription Inhibition by Fidaxomicin (Lipiarmycin A3). Mol Cell 70, 60-71 e15, doi:10.1016/j.molcel.2018.02.026 (2018).

24 Boyaci, H. et al. Fidaxomicin jams Mycobacterium tuberculosis RNA polymerase motions

25 Kohler, R., Mooney, R. A., Mills, D. J., Landick, R. \& Cramer, P. Architecture of a transcribingtranslating expressome. Science 356, 194-197, doi:10.1126/science.aal3059 (2017).

26 Pani, B. \& Nudler, E. Mechanistic insights into transcription coupled DNA repair. DNA Repair (Amst) 56, 42-50, doi:10.1016/j.dnarep.2017.06.006 (2017).

27 Lang, K. S. \& Merrikh, H. The Clash of Macromolecular Titans: Replication-Transcription Conflicts in Bacteria. Annu Rev Microbiol 72, 71-88, doi:10.1146/annurev-micro-090817062514 (2018). Harden, T. T. et al. Alternative transcription cycle for bacterial RNA polymerase. Nat Commun 11, 448, doi:10.1038/s41467-019-14208-9 (2020).

512 
Bacterial strains and plasmids

\begin{tabular}{|c|c|c|c|}
\hline & Strain & Description & Source \\
\hline \multicolumn{4}{|l|}{ E. coli } \\
\hline RNAP Msm & LK1853 & & 1 \\
\hline SigA Msm & LK1740 & $\begin{array}{l}\text { pET22b+ with C-terminal } \\
6 x \text { His SigA Msm BL21(DE3) }\end{array}$ & This work \\
\hline HelD Msm & Mshe1 & $\begin{array}{l}\text { 6xHis-HelD Msm, Lemo21 } \\
\text { (DE3) }\end{array}$ & This work \\
\hline $\mathrm{RbpA}$ Msm & LK1254 & $\begin{array}{c}\text { pET22b+ with C-terminal } \\
6 x H i s \text { RbpA Msm, } \\
\text { BL21(DE3) }\end{array}$ & This work \\
\hline \multicolumn{4}{|c|}{ M. smegmatis } \\
\hline wt & LK865 & M. smegmatis $\mathrm{mc}^{2} 155$ & Laboratory strain \\
\hline RNAP-FLAG & $\begin{array}{l}\text { LK1468 } \\
\text { MR-sspB }\end{array}$ & $\begin{array}{c}\text { kindly provided by D. } \\
\text { Schnappinger, Weill } \\
\text { Cornell Medical College, } \\
\text { New York, USA }\end{array}$ & 2 \\
\hline RbpA-FLAG & LK2541 & & This work \\
\hline SigA-FLAG & LK2073 & & This work \\
\hline HelD-FLAG & LK2590 & & This work \\
\hline
\end{tabular}

\begin{tabular}{|l|l|l|}
\hline Primer & Sequence $\mathbf{5}^{\prime} \boldsymbol{\rightarrow}^{\mathbf{3}^{\prime}}$ & \\
\hline$\# 1101$ & AAATCGGGCGGCGTCCCGGA & $\begin{array}{l}\text { Primers for Msm DNA } \\
\text { fragment for EMSA } \\
\text { assays }\end{array}$ \\
\hline$\# 1146$ & ACGGAAGCTTGGCGAGGC & $\begin{array}{l}\text { Primers for } \\
\text { (MSMEG_2758) cloning } \\
\text { into pET22b }\end{array}$ \\
\hline$\# 1155$ & GCA & \\
\hline
\end{tabular}




\begin{tabular}{|c|c|c|}
\hline \#1182 & CCGCTCGAGGCTTCCGGTTCCGCGCCG & \multirow{2}{*}{$\begin{array}{l}\text { Primers for rbpA } \\
\text { (MSMEG_3858) cloning } \\
\text { into } p E T 22 b\end{array}$} \\
\hline \#1183 & $\begin{array}{l}\text { GGAATTCCATATGATGGCTGATCGTGTCC } \\
\text { TG }\end{array}$ & \\
\hline \#2339 & $\begin{array}{l}\text { CTTCATATGGCAGCGACAAAGGCAAGCC } \\
\text { CG }\end{array}$ & \multirow{2}{*}{$\begin{array}{l}\text { Primers for } \sigma^{A} \\
\text { (MSMEG_2758) cloning } \\
\text { into pTet_INT }\end{array}$} \\
\hline \#2340 & $\begin{array}{l}\text { CGTAAGCTTCTACTTGTCGTCGTCGTCCTT } \\
\text { GTAGTCCAGGTAGTCGCGCAGCAC }\end{array}$ & \\
\hline \#2894 & $\begin{array}{l}\text { ATTCCATATGGCTGATCGTGTCCTGCGGG } \\
\text { GC }\end{array}$ & \multirow{2}{*}{$\begin{array}{l}\text { Primers for rbpA } \\
\text { (MSMEG_3858) cloning } \\
\text { into pTet_INT }\end{array}$} \\
\hline \#3093 & $\begin{array}{l}\text { CGTAAGCTTCTACTTGTCGTCGTCGTCCTT } \\
\text { GTAGTCGCTTCCGGTTCCGCGCCGCTT }\end{array}$ & \\
\hline \#3130 & $\begin{array}{l}\text { CATTCATATGTCAGGTCGGGACTACGAG } \\
\text { GAC }\end{array}$ & \multirow{2}{*}{$\begin{array}{l}\text { Primers for helD } \\
\text { (MSMEG_2174) cloning } \\
\text { into pTet_INT }\end{array}$} \\
\hline \#3131 & $\begin{array}{l}\text { CGTAAGCTTCTACTTGTCGTCGTCGTCCTT } \\
\text { GTAGTCTGCCGACGTGCGCTGCTCGACC } \\
\text { GT }\end{array}$ & \\
\hline
\end{tabular}

\section{Strain construction}

$\sigma^{A}$ (MSMEG_2758) and rbpA (MSMEG_3858) genes were amplified from genomic DNA by PCR with Phusion High-Fidelity DNA Polymerase (NEB) with primers \#1155 +\#1156 $\left(\sigma^{A}\right)$ and \#1182 + \#1183 (RbpA) and Msm chromosomal DNA as the template, cloned into pET22b via $\mathrm{Ndel} / \mathrm{Xhol}$ restriction sites and verified by sequencing. Resulting plasmids were transformed into expression Eco BL21(DE3) strain resulting in strains LK1740 $\left(\sigma^{A}\right)$ and LK1254 (RbpA).

HelD

526 Plasmid encoding the N-terminally His-tagged Msm HelD protein was prepared by the 527 GeneArt ${ }^{\circledR}$ Plasmid Construction Service (Thermofisher). Gene construct for HelD expression 528 was designed by codon-optimized back translation of gene MSMEG_2174 from Msm (strain 529 ATCC $700084 / \mathrm{mc}^{2}$ 155) with cleavage site for TEV protease placed at the $5^{\prime}$ end. This 530 synthetized gene was cloned into the Champion ${ }^{\mathrm{TM}}$ pET302/NT-His expression vector 531 (Thermofisher) via EcoRI and Xhol restriction sites. Resulting protein thus has $6 \mathrm{xHis}$ tag at its 
$\mathrm{N}$-terminus which is cleavable by TEV protease (protein construct starts with sequence MHHHHHHVNSLEENLYFQG followed by the second amino acid of gene MSMEG_2174.

\section{HelD-FLAG, $\sigma^{A}$-FLAG and RbpA-FLAG}

The genes coding for the HeID-FLAG, $\sigma^{A}-$ FLAG and RbpA-FLAG proteins were prepared by PCR using Q5 ${ }^{\circledR}$ High-Fidelity DNA Polymerase (NEB) with primers \#3130 + \#3131 (HelD), \#2339 + $\# 2340\left(\sigma^{A}\right)$ and \#2894 + \#3093 (RbpA) and Msm chromosomal DNA as the template. The Cterminal 1x FLAG-tags (DYKDDDDK) were encoded within the reverse PCR primers for all proteins. Subsequently, the genes were inserted into integrative plasmid pTet_INT (PMID: 25711368) via Ndel/HindIII restriction sites. The constructs were verified by sequencing. Resulting plasmids were transformed into Msm mc 155 (wt, LK865) cells by electroporation resulting in strains LK2590 (HelD-FLAG), LK2073 ( $\left.\sigma^{A}-F L A G\right)$ and LK2541 (RbpA-FLAG).

\section{Growth conditions}

Msm strains - mc 155 (wt, LK865), $\sigma^{\mathrm{A}}$-FLAG (LK2373), RNAP-FLAG (LK1468), HeID-FLAG (LK2590), and RbpA-FLAG (LK2541) were grown at $37^{\circ} \mathrm{C}$ in Middlebrook 7H9 medium with $0.2 \%$ glycerol and $0.05 \%$ Tween 80 and harvested in exponential phase $\left(\mathrm{OD}_{600} \sim 0.5 ; 6 \mathrm{~h}\right.$ of cultivation) or early stationary phase $\left(\mathrm{OD}_{600} \sim 2.5-3.0,24 \mathrm{~h}\right.$ of cultivation) unless stated otherwise. When required, media were supplemented with kanamycin $(20 \mu \mathrm{g} / \mathrm{ml})$. Expression in exponential phase of HelD-FLAG was induced by anhydrotetracycline $(1 \mathrm{ng} / \mathrm{ml})$ at $3 \mathrm{~h}$ of cultivation. The cells were then grown for additional $3 \mathrm{~h}$. Expressions in stationary phase of $\sigma^{A}-F L A G, R b p A-F L A G$, and HelD-FLAG were induced by anhydrotetracycline $(10 \mathrm{ng} / \mathrm{ml})$ at $8 \mathrm{~h}$ of cultivation. The cells were then cultivated for additional $16 \mathrm{~h}$.

\section{Electron microscopy}

\section{Msm RNAP core purification for cryo-EM}

Eco strain BL21(DE3) was transformed with pRMS4 (kanR) plasmid derivative encoding Msm subunits $\omega, \alpha$, and $\beta$ - $\beta^{\prime}$ fusion with C-terminal His8 tag in one operon from T7 promoter. Expression cultures were incubated at $37^{\circ} \mathrm{C}$ and shaken at $250 \mathrm{rpm}$ until OD600 $\sim 0.8$, expression was induced with $500 \mu \mathrm{M}$ isopropyl $\beta$-D-thiogalactoside (IPTG) at $17{ }^{\circ} \mathrm{C}$ for 16 h. Cells were lysed using sonication by Sonic Dismembrator Model 705 (Fisher Scientific) in a lysis buffer containing $50 \mathrm{mM} \mathrm{NaH}{ }_{2} \mathrm{PO}_{4} / \mathrm{Na}_{2} \mathrm{HPO}_{4} \mathrm{pH} 8\left(4^{\circ} \mathrm{C}\right), 300 \mathrm{mM} \mathrm{NaCl}$, 
561

562

563

564

565

566

567

568

569

570

571

572

573

574

575

576

577

578

579

580

581

582

583

584

585

586

587

588

589

$2.5 \mathrm{mM} \mathrm{MgCl}_{2}, 30 \mathrm{mM}$ imidazole, $5 \mathrm{mM} \beta$-mercaptoethanol, EDTA-free protease inhibitor cocktail (Roche), RNase A (Sigma), DNase I (Sigma) and Lysozyme (Sigma). Clarified lysate was loaded onto a HisTrap FF Crude column (GE Healthcare) and proteins were eluted with a linear gradient of imidazole to the final concentration of $400 \mathrm{mM}$ over 20 column volumes. The Msm RNAP core elution fractions were pooled and dialyzed to $20 \mathrm{mM}$ Tris$\mathrm{HCl} \mathrm{pH} 8\left(4{ }^{\circ} \mathrm{C}\right), 1 \mathrm{M} \mathrm{NaCl}, 5 \%(\mathrm{v} / \mathrm{v})$ glycerol and $4 \mathrm{mM}$ dithiothreitol (DTT) for $20 \mathrm{~h}$. The protein was further polished on XK 26/70 Superose 6 pg column (GE Healthcare) equilibrated in $20 \mathrm{mM}$ Tris- $\mathrm{HCl} \mathrm{pH} 8\left(4^{\circ} \mathrm{C}\right), 300 \mathrm{mM} \mathrm{NaCl}, 5 \%(\mathrm{v} / \mathrm{v})$ glycerol and $4 \mathrm{mM}$ DTT. The Msm RNAP core final fractions were eluted at $6 \mu \mathrm{M}$ concentration, aliquoted, flashfrozen in liquid nitrogen and then stored at $-80^{\circ} \mathrm{C}$.

\section{Msm HelD protein purification for cryo-EM}

Eco strain Lemo 21 (DE3) was transformed with pET302/NT-His (cm/R and ampR) plasmid derivative encoding the Msm HeID protein fusion with $\mathrm{N}$-terminal 6xHis tag under the control of the T7 promoter. Expression cultures were incubated at $37^{\circ} \mathrm{C}$ and shaken at 250 rpm until OD600 0.8, expression was induced with $500 \mu \mathrm{M}$ IPTG at $17{ }^{\circ} \mathrm{C}$ for $16 \mathrm{~h}$. Cells were lysed using sonication by Sonic Dismembrator Model 705 (Fisher Scientific) in a lysis buffer containing $50 \mathrm{mM}$ Tris- $\mathrm{HCl} \mathrm{pH} 7.5\left(4{ }^{\circ} \mathrm{C}\right), 400 \mathrm{mM} \mathrm{NaCl}, 30 \mathrm{mM}$ imidazole, $0.2 \%$ Tween20, 2 mM $\beta$-mercaptoethanol, EDTA-free protease inhibitor cocktail (Roche), RNase A (Sigma), DNase I (Sigma) and Lysozyme (Sigma). Clarified lysate was loaded onto a HisTrap FF Crude column (GE Healthcare) and proteins were eluted with a linear gradient of imidazole to the final concentration of $400 \mathrm{mM}$ over 20 column volumes. Fractions containing HelD protein were pooled and dialyzed for $20 \mathrm{~h}$ against the dialysis buffer containing $20 \mathrm{mM}$ Tris- $\mathrm{HCl}$, pH $7.5\left(4{ }^{\circ} \mathrm{C}\right), 500 \mathrm{mM} \mathrm{NaCl}, 1 \mathrm{mM}$ DTT together with TEV protease at a TEV protease:HeID ratio 1:20.

The protein was then concentrated to $15 \mathrm{~A}_{280}$ units and further purified using sizeexclusion chromatography using a Superdex 75 column (GE Healthcare) equilibrated in 20 mM Tris- $\mathrm{HCl}$, pH $7.5\left(4{ }^{\circ} \mathrm{C}\right), 200 \mathrm{mM} \mathrm{NaCl}$ and $1 \mathrm{mM}$ DTT. The HelD protein was eluted at $\sim 160 \mu \mathrm{M}$ concentration, aliquoted, flash-frozen in liquid nitrogen and then stored at -80 ${ }^{\circ} \mathrm{C}$. 
592

593

594

595

596

597

598

599

600

601

602

603

604

605

606

607

608

609

610

611

612

613

614

615

616

617

618

619

To assemble the HelD-RNAP complex, the individual proteins were mixed at a molar ratio of $3: 1$. The in vitro reconstitutions were carried out at $4{ }^{\circ} \mathrm{C}$, and the reconstitution mixture was incubated for $15 \mathrm{~min} .50 \mu \mathrm{l}$ of the reconstitution mixture was injected onto a Superose 6 Increase 3.2/300 column (GE Healthcare) equilibrated in $20 \mathrm{mM}$ Tris $-\mathrm{HCl}, \mathrm{pH} 7.8\left(4^{\circ} \mathrm{C}\right.$ ), $150 \mathrm{mM} \mathrm{NaCl}, 10 \mathrm{mM} \mathrm{MgCl}_{2}$ and $1 \mathrm{mM} \mathrm{DTT.} \mathrm{50- \mu l} \mathrm{fractions} \mathrm{were} \mathrm{collected} \mathrm{and} \mathrm{the} \mathrm{protein}$ was eluted at $\sim 1 \mu \mathrm{M}$ concentration.

\section{Electron microscopy}

Complexes were diluted to $\sim 850 \mathrm{nM}$ and aliquots of $3 \mu$ l were applied to Quantifoil R1.2/1.3 or $\mathrm{R} 2 / 2 \mathrm{Au} 300$ mesh grids, immediately blotted for $2 \mathrm{~s}$ and plunged into liquid ethane using an FEI Vitrobot IV $\left(4{ }^{\circ} \mathrm{C}, 100 \%\right.$ humidity).

The grids were loaded into an FEI Titan Krios electron microscope at the European Synchrotron Radiation Facility (ESRF) (beamline CM01, ESRF) or CEITEC (Masaryk University, Brno), operated at an accelerating voltage of $300 \mathrm{keV}$ and equipped with a postGIF K2 Summit direct electron camera (Gatan) operated in counting mode. Cryo-EM data was acquired using EPU software (FEI) at a nominal magnification of $\times 165,000$, with a pixel size of 0.8311 and $0.840 \AA$ per pixel. Movies of a total fluence of $\sim 40-50$ electrons per $\AA^{2}$ were collected at $\sim 1 \mathrm{e}^{-} / \AA^{2}$ per frame. A total number of 15,177 movies were acquired at a defocus range from -0.7 to $-3.3 \mu \mathrm{m}$ (Table S2).

\section{Cryo-EM image processing}

All movie frames from three datasets were aligned and dose-weighted using the MotionCor2 program (Figure S3a) and then used for contrast transfer function parameter calculation with $\mathrm{Gctf}^{3}$. Initially, particles were selected without a template by Gautomatch (provided by Dr. Kai Zhang, http://www.mrc-Imb.cam.ac.uk/kzhang) from a small portion of the data set ( 200 movies). This initial small dataset was subjected to reference-free 2D-classification using RELION 3.04. Eight representative classes of different views were selected from the twodimensional averages and used as reference for automatic particle picking for the dataset I by RELION. WARP ${ }^{5}$ was used for particle picking for datasets II and III. 
620

621

622

623

624

625

626

627

628

629

630

631

632

633

634

635

636

637

638

639

640

641

642

643

644

645

646

647

648

649

(Figure S3b) at $3 \mathrm{x}$ and $2 \mathrm{x}$ binned a pixel size. Particles in classes with poor structural features were removed. Particles from dataset I and II were globally refined to estimate the pixel size matching ${ }^{6}$ and particles from dataset II were estimated to match the common pixel size $0.8311 \AA$ A per pixel. Particles from all datasets were pooled ( 1,560 k), 2x binned and subjected to three-dimensional classifications with image alignment (Figure S4). The first round of 3Dclassification was restricted to ten classes and performed using Msm RNAP core (PDB entry 6 F6W) as a $60 \AA$ low-pass filtered initial model. Classification was done during three rounds of 25 iterations each, using regularization parameter $T=4$. During the second and third round, local angular searches were performed at $3.5^{\circ}$ and $1.8^{\circ}$ to clearly separate structural species. The three most abundant and defined 3D-classes were re-extracted at the pixel size of $0.8311 \AA$ per pixel and 3D auto-refined using respective masks in RELION 3.0 (Figure S4). The results of the 3D auto-refinement were used for per particle CTF refinement in RELION 3.1 ${ }^{7}$ and further 3D auto-refined. Further 3D classification was applied on class 1 and 3, but no better defined 3D classes were identified. The 3D reconstruction of class 2 was further focus 3D auto-refined on the RNAP core region. The 3D reconstruction of class 2 was also 3D focus classified on the region of the HelD-specific domain and a more defined class was identified and 3D auto-refined separately. The final cryo-EM density maps were generated by the post-processing feature in RELION and sharpened or blurred into MTZ format using $\mathrm{CCP}-\mathrm{EM}^{8}$. The resolutions of the cryo-EM density maps were estimated at the 0.143 gold standard Fourier Shell Correlation (FSC) cut off (Figure S3e). A local resolution (Figure S3d) was calculated using RELION and reference-based local amplitude scaling was performed by LocScale ${ }^{9}$.

\section{Cryo-EM model building and refinement}

Atomic models of Msm RNAP protein parts (Figure 1a-c) were generated according to the known structure of the Msm RNAP core (PDB entry 6F6W). The whole RNAP core was first rigid-body fitted into the cryo-EM density by Molrep ${ }^{10}$ and individual sub-domains fits were optimized using the Jigglefit tool ${ }^{11}$ in $\operatorname{Coot}^{\underline{12}}$ and best fits were chosen according to a correlation coefficient in the JiggleFit tool. The crystal structure of the Bsu HelD-2A domain (Figure S7g) was first rigid-body fitted into the cryo-EM density by Molrep ${ }^{10}$ and then manually adapted in Coot. Parts of the HelD main chain were first traced into the cryo-EM 
650

651

652

653

654

655

656

657

658

659

660

661

662

663

664

665

666

667

668

669

670

671

672

673

674

675

676

677

678

679

density by Buccaneer ${ }^{13}$ and Mainmast ${ }^{14}$.The rest of the HelD protein was built de-novo in Coot $^{11}$. The cryo-EM atomic-models of HelD-RNAP complexes were then iteratively improved by manual building in Coot and refinement and validation with Phenix real-space refinement ${ }^{15}$. The atomic models were validated with the Phenix validation tool (Table S2) and the model resolution was estimated at the 0.5 FSC cut-off. Structures were analyzed and Figures were prepared using the following software packages: PyMOL (Schrödinger, Inc.) with APBS plugin ${ }^{16}$, USCF Chimera ${ }^{17}$, CCP4MG $^{18}$, ePISA server ${ }^{19}$.

\section{X-ray crystal structure determination of the Bsu HelD C-terminal domain}

DNA sequence encoding the C-terminal domain of HelD (from residue 608 to 774) was amplified by PCR and cloned into $\mathrm{pET15b}$ vector by $\mathrm{Ndel}$ and $\mathrm{BamHI}$ restriction sites to make an N-terminal His6-tagged protein. Bacterial culture containing BL21(DE3) RIPL codon-plus cells transformed with a pET15b-HelD-CTD vector was grown at $37{ }^{\circ} \mathrm{C}$ in LB medium supplemented with $100 \mu \mathrm{g} / \mathrm{ml}$ ampicillin, protein expression was induced with $0.5 \mathrm{mM}$ IPTG at $\mathrm{OD}_{600}=0.5$, and incubated for additional $3 \mathrm{~h}$ to allow protein expression. Cells were harvested by centrifugation and lysed by sonication in lysis buffer $(50 \mathrm{mM}$ Tris- $\mathrm{HCl}, \mathrm{pH} 8.0$ at $4{ }^{\circ} \mathrm{C}, 200 \mathrm{mM} \mathrm{NaCl}$, 5\% glycerol, $2 \mathrm{mM} \beta$-mercaptoethanol, $2 \mathrm{mM}$ phenylmethylsulfonyl fluoride, PMSF). The lysate was clarified by centrifugation and HeID-CTD was purified by NiNTA, Q-sepharose and Heparin-column chromatography. Fractions containing HelD-CTD were concentrated using VivaSpin concentrators until $10 \mathrm{mg} / \mathrm{ml}$ in crystallization buffer $(10 \mathrm{mM}$ Tris- $\mathrm{HCl}, \mathrm{pH} 8$ at $4{ }^{\circ} \mathrm{C}, 50 \mathrm{mM} \mathrm{NaCl}, 1 \%$ glycerol, $0.1 \mathrm{mM}$ EDTA, $1 \mathrm{mM} \mathrm{DTT).}$

Crystallization condition of HelD-CTD was screened by using JCSG+ screen (Molecular Dimensions) and crystals were obtained in crystallization solution (0.1 M Na/K phosphate, $\mathrm{pH}$ $6.2,0.2 \mathrm{M} \mathrm{NaCl}, 50 \%$ PEG200) at $22^{\circ} \mathrm{C}$. X-ray crystallographic data were collected at the Penn State X-ray Crystallography Facility and the data were processed by $\mathrm{HKL} 2000^{20}$. For Sulfur single-wavelength anomalous dispersion phasing, $10 \mathrm{~S}$ atom positions were identified and the initial phase and density-modified map were calculated by AutoSol followed by automated model building by AutoBuild in the program Phenix ${ }^{15}$. Iterative refinement by Phenix and model building using $\operatorname{Coot}^{12}$ improved the map and model. Finally, water molecules were added to the model. The data statistics and X-ray structure parameters are shown in Table S3. 


\section{Protein purification for biochemical assays}

682

683

684

685

686

687

688

689

690

691

692

693

694

695

696

697

698

699

700

701

702

703

704

705

706

707

\section{Msm RNAP core}

Strain of Eco containing plasmid with subunits of the RNAP core $\left(\right.$ LK1853 ${ }^{1}$ ) was grown to the exponential phase $\left(\mathrm{OD}_{600} \sim 0.5\right)$. Expression of RNAP was induced with $500 \mu \mathrm{M}$ IPTG for $4 \mathrm{~h}$ at room temperature. Cells were harvested by centrifugation, washed, resuspended in P buffer (300 mM NaCl, 50 mM Na $2 \mathrm{HPO}_{4}$, 5\% glycerol, $3 \mathrm{mM} \beta$-mercaptoethanol) and disrupted by sonication. Cell debris was removed by centrifugation and supernatant was mixed with $1 \mathrm{ml}$ Ni-NTA Agarose (Qiagen) and incubated for 90 minutes at $4{ }^{\circ} \mathrm{C}$ with gentle shaking. Ni-NTA Agarose with bound RNAP was loaded on a Poly-Prep ${ }^{\circledR}$ Chromatography Column (BIO-RAD), washed with P buffer and, subsequently, washed with $\mathrm{P}$ buffer with $30 \mathrm{mM}$ imidazole. The proteins were eluted with $\mathrm{P}$ buffer containing $400 \mathrm{mM}$ imidazole and fractions containing RNAP were pooled and dialysed against storage buffer (50 mM Tris- $\mathrm{HCl}, \mathrm{pH} 8.0,100 \mathrm{mM} \mathrm{NaCl}$, $50 \%$ glycerol, $3 \mathrm{mM} \beta$-mercaptoethanol). The RNAP protein was stored at $-20^{\circ} \mathrm{C}$.

\section{Msm $\sigma^{A}$}

Expression strain of Eco containing plasmid with gene of $\sigma^{\mathrm{A}}$ (LK1740, this work) was grown at $37^{\circ} \mathrm{C}$ until $\mathrm{OD}_{600}$ reached $\sim 0.5$; expression of $\sigma^{A}$ was induced with $300 \mu \mathrm{M}$ IPTG at room temperature for $3 \mathrm{~h}$. Isolation of $\sigma^{A}$ was done in the same way as RNAP purification with the exception of $50 \mathrm{mM}$ imidazole added to the $\mathrm{P}$ buffer before resuspending the cells. Instead of the purification in a column, batch purification and centrifugation were used to separate the matrix and the eluate.

\section{Msm RbpA}

The expression and purification of RbpA (LK1254, this work) were done in the same way as for RNAP except when $\mathrm{OD}_{600}$ reached $\sim 0.5$, the expression was induced with $800 \mu \mathrm{M}$ IPTG at room temperature for $3 \mathrm{~h}$.

\section{Msm HelD}

Msm HelD was prepared as described previously, in the paragraph about purification of proteins for cryo-EM experiments. 
Purity of all purified proteins was checked by SDS-PAGE gel.

Hydrolysis of ATP, GTP and CTP (Sigma-Aldrich) by Msm HelD was measured in a total volume of $50 \mu \mathrm{l}$ reaction mixture which contained $10 \mathrm{mM}$ substrate, $10 \mu \mathrm{g}$ of $\mathrm{Msm}$ HelD and reaction buffer composed of $50 \mathrm{mM}$ Tris- $\mathrm{HCl}, \mathrm{pH} 7.5,50 \mathrm{mM} \mathrm{NaCl}, 5 \mathrm{mM} \mathrm{MgCl}_{2}$. Incubation was carried out at $37{ }^{\circ} \mathrm{C}$ for $30 \mathrm{~min}$. The amount of released phosphate was analyzed spectrophotometrically at $\lambda=850 \mathrm{~nm}$ according to a modified molybdenum blue method ${ }^{21}$ using a microplate reader Clariostar (BMG LABTECH, Ortenberg, Germany). Briefly, the reaction was stopped by adding $62 \mu \mathrm{l}$ of reagent $\mathrm{A}\left(0.1 \mathrm{M} \mathrm{L}\right.$-ascorbic acid, $\left.0.5 \mathrm{M} \mathrm{Cl}_{3} \mathrm{CCOOH}\right)$. After thorough mixing, $12.5 \mu$ l of reagent $\mathrm{B}\left(10 \mathrm{mM}\left(\mathrm{NH}_{4}\right)_{6} \mathrm{Mo}_{7} \mathrm{O}_{24}\right)$ and $32 \mu \mathrm{l}$ of reagent $\mathrm{C}(0.1$ $\mathrm{M}$ sodium citrate, $0.2 \mathrm{M} \mathrm{NaAsO}_{2}, 10 \%$ acetic acid) was added. All enzymatic reactions were performed in triplicates with separate background readings for each condition.

\section{DNA-Protein interaction analysis in vitro}

DNA-Protein interactions were analyzed on $4-16 \%$ Bis-Tris native gels (Thermo Fisher Scientific, cat. No. BN1002BOX) by Electrophoretic Mobility Shift Assay (EMSA). DNA fragment was amplified by Expand High Fidelity PCR System (Roche, cat. No. 11732650001) using \#1101 and \#1146 primers and Msm chromosomal DNA. The resulting 304 bp long PCR fragment was excised and purified from agarose gel. Binding reactions were performed in 1xSTB buffer (50 $\mathrm{mM}$ Tris- $\mathrm{HCl} \mathrm{pH} 8.0 ; 5 \mathrm{mM} \mathrm{Mg}\left(\mathrm{C}_{2} \mathrm{H}_{3} \mathrm{O}_{2}\right) 2 ; 100 \mu \mathrm{M}$ DTT; $50 \mathrm{mM} \mathrm{KCl} ; 50 \mu \mathrm{g} / \mathrm{ml} \mathrm{BSA}$ ) that contained RNAP (25 pmol), HelD (125 pmol) and DNA (0.2 pmol). First, RNAP was preincubated in the presence or absence of HelD (at $37^{\circ} \mathrm{C}, 45 \mathrm{~min}$ ). Subsequently, DNA was added and samples were incubated at $37{ }^{\circ} \mathrm{C}$ for additional $45 \mathrm{~min}$. Then, NativePage buffer (Invitrogen, cat. No. BN2003) was added and samples were loaded on native gel. Electrophoresis was run in cold room $\left(4^{\circ} \mathrm{C}\right)$. Finally, the gel was stained with DNA stain GelRed nucleic acid stain (Biotium, cat. No. 41003) in 1xTBS for 25 minutes and images were taken with an Ingenius UV-light camera (Syngen). Unbound DNA was quantified by the Quantity One software (BioRad). The gel was subsequently stained with Simply Blue (Invitrogen, cat. No. LC6060) for protein visualization. 


\section{Protein-Protein interaction analysis in vitro}

Protein-Protein interactions were analyzed on 7\% Tris-acetate native gels (Thermo Fisher Scientific, cat. No. EA0355BOX) by EMSA. Binding reaction was done in $20 \mu \mathrm{l}$ of 1xSTB buffer containing RNAP (25 pmol), HelD (125 pmol), $\sigma^{A}$ (1,250 pmol) and RbpA (1,250 pmol) - protein combinations in reactions are specified in the Figure 4 legend. First, RNAP was reconstituted with/without HelD (at $37{ }^{\circ} \mathrm{C}, 45 \mathrm{~min}$ ). Then $\mathrm{RbpA}$ and/or $\sigma^{\mathrm{A}}$ were added, followed by additional incubation at $37^{\circ} \mathrm{C}$ for $45 \mathrm{~min} .20 \mu$ of Native Tris-Glycine buffer (Invitrogen, cat. No. LC2673) was added and $20 \mu \mathrm{l}$ of the mixture was then loaded on a native gel. Electrophoresis was run in cold room $\left(4^{\circ} \mathrm{C}\right)$. Subsequently, for protein visualization, the gels were stained with Simply Blue. The identity of proteins in each band was determined by MALDI mass spectrometric identification.

\section{Disassembly of elongation complexes}

Elongation complexes (ECs), containing a transcription bubble, were assembled with the Msm RNAP core as described before ${ }^{22}$. DNA and RNA oligonucleotides were purchased and are the same as in Table EV7 in ${ }^{23}$. The RNA (LK-pRNA) was monophosphorylated at the $5^{\prime}$ end by the manufacturer. A 2-fold molar excess of RNA was mixed with template DNA (LK632) in water and annealed in a cycler $\left(45^{\circ} \mathrm{C}\right.$ for $2 \mathrm{~min}, 42-27^{\circ} \mathrm{C}$ : temperature was decreasing by $3^{\circ} \mathrm{C}$ every $2 \mathrm{~min}, 25^{\circ} \mathrm{C}$ for $10 \mathrm{~min}$ ). RNAP (32 pmol per sample) was incubated with 4 pmol of the annealed hybrid in $10 \mu$ l of reaction buffer ( $40 \mathrm{mM}$ Tris- $\mathrm{HCl}$, pH 8.0, $10 \mathrm{mM} \mathrm{MgCl}, 1 \mathrm{mM}$ DTT) for $15 \mathrm{~min}$ at room temperature with gentle shaking. 8 pmol of non-template DNA (LK631) containing biotin at the $5^{\prime}$ end was added and the mixture was incubated at $37^{\circ} \mathrm{C}$ for $10 \mathrm{~min}$.

Streptavidin-coated magnetic beads ( $25 \mu$ l per sample; Sigma S-2415) were washed with 500 $\mu \mathrm{l}$ of binding buffer $(20 \mathrm{mM}$ Tris- $\mathrm{HCl}, \mathrm{pH} 8.0,0.15 \mathrm{M} \mathrm{NaCl})$ and resuspended in the same volume of fresh binding buffer. Assembled elongation complexes were then mixed with washed beads. ECs and beads were incubated together for 30 min at RT (room temperature) with continuous gentle shaking. Unbound complexes were removed by subsequent washing with $500 \mu \mathrm{l}$ of binding buffer, $500 \mu$ l of washing buffer $(20 \mathrm{mM}$ Tris- $\mathrm{HCl} \mathrm{pH} 8.0,0.5 \mathrm{M} \mathrm{NaCl}, 2$ $\mathrm{mM} \mathrm{MgCl} 2,1 \mathrm{mM} \mathrm{DTT}$ ) and $500 \mu \mathrm{l}$ of reaction buffer ${ }^{24}$. Beads were resuspended in reaction buffer with $100 \mathrm{mM}$ final concentration of $\mathrm{KCl}$, with or without GTP or ATP (final concentration $200 \mu \mathrm{M}$ ) in a total volume of $5 \mu \mathrm{l}$. HelD in 2 -fold ratio over RNAP (64 pmol per sample) or 
heat-inactivated HelD $\left(5 \mathrm{~min}\right.$ at $\left.95^{\circ} \mathrm{C}\right)$ or buffer were added to the final reaction volume of $10 \mu \mathrm{l}$. Reactions proceeded for $20 \mathrm{~min}$ at $37^{\circ} \mathrm{C}$. The bound (in complex with EC) and released (free in buffer) RNAPs were separated by using a DYNAL Invitrogen bead separation device. Subsequently, the fractions containing released RNAPs were spotted directly on nitrocellulose membrane. RNAPs were detected by Western blotting using mouse monoclonal antibodies against the $\beta$ subunit of RNAP (clone name 8RB13) and secondary antibodies conjugated with a fluorophore dye (WesternBrightTM MCF-IR, Advansta, $800 \mathrm{~nm}$ anti-mouse antibody) and scanned with an Odyssey reader (LI-COR Biosciences). The analysis was done with the Quantity One software (BioRad). The experiment was conducted in five biological replicates.

\section{Immunoprecipitation}

$150 \mathrm{ml}$ of Msm exponential (Figure S9) and $100 \mathrm{ml}$ of stationary phase (Figures 4f, S1, S10) cells were pelleted and resuspended in $4 \mathrm{ml}$ of Lysis buffer $(20 \mathrm{mM}$ Tris- $\mathrm{HCl}, \mathrm{pH} 8,150 \mathrm{mM}$ $\mathrm{KCl}, 1 \mathrm{mM} \mathrm{MgCl}$ ) with $1 \mathrm{mM} \mathrm{DTT}, 0.5 \mathrm{mM} \mathrm{PMSF}$ and Sigma protease inhibitor cocktail P8849 $(5 \mu \mathrm{l} / \mathrm{ml})$, sonicated $15 \times 10 \mathrm{~s}$ with $1 \mathrm{~min}$ pauses on ice and centrifuged. $1 \mathrm{ml}$ of stationary and $1.5 \mathrm{ml}$ of exponential phase cells lysates were incubated over night at $4{ }^{\circ} \mathrm{C}$ with $25 \mu \mathrm{l}$ of ANTIFLAG $^{\circledR}$ M2 Affinity Agarose Gel (Sigma, A2220). Agarose gel beads with the captured protein complexes were washed $4 x$ with $0.5 \mathrm{ml} 20 \mathrm{mM}$ Tris- $\mathrm{HCl}, \mathrm{pH} 8,150 \mathrm{mM} \mathrm{KCl}, 1 \mathrm{mM} \mathrm{MgCl}$. FLAG-tagged proteins were eluted by $60 \mu \mathrm{l}$ of $3 \times$ FLAG $^{\circledR}$ Peptide (Sigma F4799) (diluted in Trisbuffered saline (TBS) to a final concentration of $150 \mathrm{ng} / \mathrm{ml}$ ). Proteins were resolved on sodium dodecylsulphate-polyacrylamide gel electrophoresis (SDS-PAGE) and Simply Blue-stained (SimplyBlue, Invitrogen) or analyzed by Western blotting.

\section{Double pull-down}

Eluted proteins from the first immunoprecipitation (ANTI-FLAG, see above) from lysates of the HelD-FLAG culture from exponential phase were incubated $\left(\mathrm{O} / \mathrm{N}, 4^{\circ} \mathrm{C}\right)$ with $5 \mu \mathrm{g}$ of $\sigma^{A}$ or IgG antibodies (negative control), respectively, bound to $20 \mu \mathrm{l}$ of Protein G-plus Agarose (Santa Cruz Biotechnology, Cat. No. sc-2002), and then 4x washed with $1 \mathrm{ml}$ Lysis buffer. Finally, proteins were analyzed by SDS-PAGE and Western blot. 


\section{Western blotting}

797 Proteins were resolved by SDS-PAGE and detected by Western blotting using mouse monoclonal antibodies against $\sigma^{70} / \sigma^{A}$ (clone name 2G10, Biolegend, cat. No. 663208), against the $\beta$ subunit of RNAP (clone name 8RB13, Biolegend, cat. No. 663903), monoclonal ANTIFLAG (clone M2, Sigma cat. No. F1814), and anti-mouse secondary antibodies conjugated with HRP (Sigma, cat. No. A7058). Subsequently, the blot was incubated for 5 min with SuperSignal ${ }^{\mathrm{TM}}$ West Pico PLUS Chemiluminiscent substrate (Thermo scientific, cat. No. 34577), exposed on film and developed.

Trypsin digestion and MALDI mass spectrometric identification

Simply Blue-stained protein bands were cut out from gels, chopped into small pieces and destained using $50 \mathrm{mM}$ 4-ethylmorpholine acetate ( $\mathrm{pH}$ 8.1) in $50 \%$ acetonitrile (MeCN). The gel pieces were then washed with water, reduced in size by dehydration in MeCN and partly dried in a SpeedVac concentrator. The proteins were digested overnight at $37{ }^{\circ} \mathrm{C}$ using sequencing grade trypsin (100 ng; Promega) in a buffer containing 25 mM 4-ethylmorpholine acetate and 5\% MeCN. The resulting peptides were extracted with 40\% MeCN/0.2\% TFA (trifluoroacetic acid).

For MALDI MS analysis, $0.5 \mu$ l of each peptide mixture was deposited on the MALDI plate, airdried at room temperature, and overlaid with $0.5 \mu \mathrm{l}$ of the matrix solution ( $\alpha$-cyano-4hydroxycinnamic acid in 50\% acetonitrile/0.1\% TFA; $5 \mathrm{mg} / \mathrm{ml}$, Sigma). Peptide mass maps of proteins in Figures 4f, S1 were measured using an Autoflex Speed MALDI-TOF instrument (Bruker Daltonics, Billerica, USA) in a mass range of 700-4000 Da and calibrated externally using a PepMix II standard (Bruker Daltonics). For protein identification, MS spectra were searched against NCBIprot_20190611 database subset of bacterial proteins using the inhouse MASCOT v.2.6 search engine with the following settings: peptide tolerance of $20 \mathrm{ppm}$, missed cleavage site set to one and variable oxidation of methionine. The spectra of proteins in Figure 4h were acquired on a 15T Solarix XR FT-ICR mass spectrometer (Bruker Daltonics) in a mass range of 500-6000 Da and calibrated internally using peptide masses of Msm RpoB and RpoC proteins. The peak lists generated using DataAnalysis 5.0 program were searched against UniProtKB database of Msm proteins using the in-house MASCOT engine with the 
following settings: peptide tolerance of $3 \mathrm{ppm}$, missed cleavage site set to two and variable and oxidation of methionine.

\section{Methods references}

8281 Kouba, T. et al. The Core and Holoenzyme Forms of RNA Polymerase from Mycobacterium smegmatis. J Bacteriol 201, doi:10.1128/JB.00583-18 (2019).

$8302 \mathrm{Kim}, \mathrm{J}$. H. et al. Protein inactivation in mycobacteria by controlled proteolysis and its application to deplete the beta subunit of RNA polymerase. Nucleic Acids Res 39, 2210-2220, doi:10.1093/nar/gkq1149 (2011).

3 Zhang, K. Gctf: Real-time CTF determination and correction. J Struct Biol 193, 1-12, doi:10.1016/j.jsb.2015.11.003 (2016).

4 Zivanov, J. et al. New tools for automated high-resolution cryo-EM structure determination in RELION-3. Elife 7, doi:10.7554/eLife.42166 (2018).

5 Tegunov, D. \& Cramer, P. Real-time cryo-electron microscopy data preprocessing with Warp. Nat Methods 16, 1146-1152, doi:10.1038/s41592-019-0580-y (2019).

6 Wilkinson, M. E., Kumar, A. \& Casanal, A. Methods for merging data sets in electron cryomicroscopy. Acta Crystallogr D Struct Biol 75, 782-791, doi:10.1107/S2059798319010519 (2019).

7 Zivanov, J., Nakane, T. \& Scheres, S. H. W. Estimation of high-order aberrations and anisotropic magnification from cryo-EM data sets in RELION-3.1. IUCrJ 7, 253-267, doi:10.1107/S2052252520000081 (2020).

8 Burnley, T., Palmer, C. M. \& Winn, M. Recent developments in the CCP-EM software suite. Acta Crystallogr D Struct Biol 73, 469-477, doi:10.1107/S2059798317007859 (2017).

9 Jakobi, A. J., Wilmanns, M. \& Sachse, C. Model-based local density sharpening of cryo-EM maps. Elife 6, doi:10.7554/eLife.27131 (2017).

10 Vagin, A. \& Teplyakov, A. Molecular replacement with MOLREP. Acta Crystallogr D Biol Crystallogr 66, 22-25, doi:10.1107/\$0907444909042589 (2010).

11 Brown, A. et al. Tools for macromolecular model building and refinement into electron cryomicroscopy reconstructions. Acta Crystallogr D Biol Crystallogr 71, 136-153, doi:10.1107/S1399004714021683 (2015).

12 Emsley, P. \& Cowtan, K. Coot: model-building tools for molecular graphics. Acta Crystallogr D Biol Crystallogr 60, 2126-2132, doi:S0907444904019158 [pii], doi 10.1107/S0907444904019158 (2004).

13 Cowtan, K. The Buccaneer software for automated model building. 1. Tracing protein chains. Acta crystallographica. Section D, Biological crystallography 62, 1002-1011, doi:10.1107/S0907444906022116 (2006).

14 Terashi, G. \& Kihara, D. De novo main-chain modeling for EM maps using MAINMAST. Nat Commun 9, 1618, doi:10.1038/s41467-018-04053-7 (2018).

15 Afonine, P. V. et al. Real-space refinement in PHENIX for cryo-EM and crystallography. Acta Crystallogr D Struct Biol 74, 531-544, doi:10.1107/S2059798318006551 (2018).

16 Jurrus, E. et al. Improvements to the APBS biomolecular solvation software suite. Protein Sci 27, 112-128, doi:10.1002/pro.3280 (2018).

17 Pettersen, E. F. et al. UCSF Chimera--a visualization system for exploratory research and analysis. J Comput Chem 25, 1605-1612, doi:10.1002/jcc.20084 (2004).

18 McNicholas, S., Potterton, E., Wilson, K. S. \& Noble, M. E. Presenting your structures: the CCP4mg molecular-graphics software. Acta Crystallogr D Biol Crystallogr 67, 386-394, doi:10.1107/S0907444911007281 (2011). 
19 Schlee, S. et al. Prediction of quaternary structure by analysis of hot spot residues in proteinprotein interfaces: the case of anthranilate phosphoribosyltransferases. Proteins 87, 815825, doi:10.1002/prot.25744 (2019).

20 Otwinowski, Z. \& Minor, W. Processing of X-ray diffraction data collected in oscillation mode. Methods Enzymol 276, 307-326 (1997).

21 He, Z. \& Honeycutt, C. W. A Modified Molybdenum Blue Method for Orthophosphate Determination Suitable for Investigating Enzymatic Hydrolysis of Organic Phosphates. Communications in Soil Science and Plant Analysis 36, 1373-1383, doi:10.1081/CSS200056954 (2005).

22 Komissarova, N., Kireeva, M. L., Becker, J., Sidorenkov, I. \& Kashlev, M. Engineering of elongation complexes of bacterial and yeast RNA polymerases. Methods Enzymol 371, 233 251, doi:10.1016/S0076-6879(03)71017-9 (2003).

23 Sikova, M. et al. The torpedo effect in Bacillus subtilis: RNase J1 resolves stalled transcription complexes. EMBO J 39, e102500, doi:10.15252/embj.2019102500 (2020).

24 Deng, Z. et al. Yin Yang 1 regulates the transcriptional activity of androgen receptor. Oncogene 28, 3746-3757, doi:10.1038/onc.2009.231 (2009).

Acknowledgments: We thank the ESRF (especially Michael Hons), IBS and EMBL for access to the ESRF Krios beamline CM01; the CEITEC and the Czech Infrastructure for Integrative Structural Biology (CIISB) for access to the CEITEC Krios microscope and to the CMS facilities at BIOCEV (project LM2015043 by MEYS). This work was supported by 20-12109S (to LK) and 20-07473S (to JH) from the Czech Science Foundation, NIH grant R35 GM131860 to KM, and by the Academy of Sciences of the Czech Republic (RVO: 86652036), MEYS (CZ.1.05/1.1.00/02.0109), European Regional Development Fund (Project CIISB4HEALTH, No. CZ.02.1.01/0.0/0.0/16_013/0001776 and ELIBIO, No. CZ.02.1.01/0.0/0.0/15_003/0000447), T Kou. holds a fellowship from the EMBL Interdisciplinary Postdocs (EI3POD) initiative cofunded by Marie Skłodowska-Curie grant agreement No. 664726.

Author contributions: JD and LK conceived and supervised the project. TKou, TKov, MT, and JDu expressed and purified proteins for cryo-EM, TKou prepared cryoEM grids, collected cryoEM data together with $\mathrm{JN}$, performed image processing and 3D reconstruction, and built 
initial models together with JDo. KSM and UC solved the Bsu HeID CTD. MJ, JH, BB, MŠ, JP, PS, and HŠ did cloning, protein purifications and IPs. JP and PS performed DNA binding experiments. MT, JDu, and TKov performed NTP hydrolysis experiments. TKou, TKov and JDo built and refined atomic models and created figures. IB and MS performed in silico modelling. TKou, TKov, JDo and LK wrote the manuscript with input from IB. TS performed initial modelling and comparative analysis.

Competing interests: The authors declare no competing interests.

Data Availability: Co-ordinates and structure factors or maps have been deposited in the wWWPDB or EMDB.

Bsu HelD C-terminal domain (X-ray) PDB ID 6VSX

Msm HelD-RNAP complex State III (cryoEM) EMD-11026, PDB ID $6 Z 11$

Movie 1: Cryo-EM structure of Msm HelD-RNAP complexes in State I - PCh-engaged engaged

917 Movie 2: Cryo-EM structure of Msm HelD-RNAP complexes in State II - PCh-engaged and AS-

918 interfering

919 Movie 3: Cryo-EM structure of Msm HelD-RNAP complexes in State III - PCh dis-engaged and 